\title{
Anomalous currents and constitutive relations of a chiral hadronic superfluid
}

\author{
Juan L. Mañes, ${ }^{a}$ Eugenio Megías, ${ }^{b, c}$ Manuel Valle ${ }^{b}$ and Miguel Á. Vázquez-Mozo ${ }^{d}$ \\ ${ }^{a}$ Departamento de Física de la Materia Condensada, Universidad del País Vasco UPV/EHU, \\ Apartado 644, 48080 Bilbao, Spain \\ ${ }^{b}$ Departamento de Física Teórica, Universidad del País Vasco UPV/EHU, \\ Apartado 644, 48080 Bilbao, Spain \\ ${ }^{c}$ Departamento de Fúsica Atómica, Molecular y Nuclear and \\ Instituto Carlos I de Física Teórica y Computacional, \\ Universidad de Granada, Avenida de Fuente Nueva s/n, 18071 Granada, Spain \\ ${ }^{d}$ Departamento de Física Fundamental, Universidad de Salamanca, \\ Plaza de la Merced s/n, 37008 Salamanca, Spain \\ E-mail: wmpmapaj@lg.ehu.es, emegias@ugr.es, manuel.valle@ehu.es, \\ Miguel.Vazquez-Mozo@cern.ch
}

ABSTRACT: The anomalous currents of two-flavor chiral nuclear matter in the presence of chiral imbalance are computed, using recently developed methods exploiting generalized transgression, which facilitates the evaluation of both the equilibrium partition function and the covariant currents. The constitutive relations for both the broken and unbroken phase of the theory are studied and the out-of-equilibrium nondissipative transport coefficients determined. In the superfluid phase, the vector covariant currents exhibit nondissipative chiral electric, magnetic, and vortical effects, the latter governed by chiral imbalance.

Keywords: Anomalies in Field and String Theories, Thermal Field Theory

ARXIV EPRINT: 1910.04013 


\section{Contents}

1 Introduction 1

2 Equilibrium partition functions from differential geometry 3

3 Two-flavor QCD fluid with unbroken chiral symmetry 11

\begin{tabular}{ll}
3.1 & Partition function and gauge currents \\
\hline
\end{tabular}

$\begin{array}{ll}3.2 & \text { Baryon, isospin, and electromagnetic currents } \\ \end{array}$

4 Pion partition function of two-flavor QCD in the chiral limit 17

5 Constitutive relations of the two-flavor hadronic superfluid $\quad 20$

$\begin{array}{ll}5.1 \text { The gauge currents at leading order } & 21\end{array}$

5.2 Covariant currents and Bardeen-Zumino terms at first order in derivatives 22

5.3 Corrections to the leading order constitutive relations 24

$\begin{array}{llr}6 & \text { Closing remarks } & 29\end{array}$

\section{Introduction}

Quantum chiral anomalies are associated with very robust mathematical properties of gauge fields beyond perturbation theory [1-6]. A practical consequence of this is that the anomalous contribution to the quantum effective action can be obtained from very general considerations using differential geometry. Although nonlocal in (Euclidean) dimension $D=2 n-2$, the effective action can be written as a local functional in $D+1=2 n-1$ dimensions, using the Chern-Simons form associated with the appropriate anomaly polynomial. For systems exhibiting spontaneous symmetry breaking, the mathematical structure of the anomaly determines the Wess-Zumino-Witten (WZW) effective Lagrangian describing the low-energy interaction of Nambu-Goldstone (NG) bosons with gauge fields.

This state of affairs turns out to be specially useful in the study of anomalous hydrodynamic transport $[7,8]$, where fluids are coupled to classical external sources through currents affected by 't Hooft anomalies. These novel class of transport phenomena include the chiral magnetic $[9,10]$ and electric [11] effects, as well as the chiral vortical effect [12] (see also $[13,14]$ for dedicated reviews). They are of physical relevance in various physical setups, including heavy ion collisions [15-18] as well as astrophysics and cosmology [19-23].

The equilibrium partition functions at finite temperature for these systems can be computed from the anomalous effective action functional by implementing dimensional reduction on the Euclidean time cycle [24]. The anomaly is then carried by the local part of the partition function while the covariant currents, giving the response of the system to 
the physical sources, can be obtained from its nonlocal piece. Transport coefficients are then read off the expressions of these currents. The application of these techniques to the study of anomalous hydrodynamics has been developed in a number of works [24-31].

In a previous paper [32], we used differential geometry to obtain explicit operative expressions allowing the computation of the equilibrium partition function of fluids in the presence of quantum ('t Hooft) anomalies, as well as the construction of the various currents associated with the corresponding global symmetries. Although applicable to generic theories, the strategy presented there turned out to be particularly powerful for systems with spontaneous symmetry breaking, where the covariant anomalous currents can be obtained without going through the study of the WZW effective action [33, 34], which generically has a rather cumbersome expression. In fact, these currents can be readily computed by an appropriate transformation of the Bardeen-Zumino (BZ) terms of the unbroken theory using the NG boson matrix. This method has been recently implemented in the study of multi-Weyl semimetals [35].

In this work we show how the results of ref. [32] are applied to the analysis of chiral nuclear matter (super)fluids in the presence of chiral, isospin, and baryon number imbalance. More specifically, we study a two-flavor hadronic fluid with unbroken chiral symmetry, as well as its superfluid phase when this symmetry is spontaneously broken. In the latter case, the equilibrium partition function is computed from the WZW functional by implementing dimensional reduction. The standard computation of the consistent currents would proceed by taking functional derivatives of this complicated action with respect to classical external sources. The corresponding covariant (vector and axial-vector) currents would then be obtained by adding the appropriate BZ terms. Instead of doing this, here we compute the covariant currents directly from the BZ polynomials using the explicit formulae found in [32], which notably simplifies the calculation.

By expressing the currents in terms of the different Lorentz tensor structures built from the NG bosons, together with the magnetic field and the vorticity, we identify the different transport phenomena present in the theory and compute the corresponding out-ofequilibrium transport coefficients. Our results show the existence of the chiral electric effect first found in [11] and confirm its nondissipative nature. In addition to this, we also find that all dependence of the electromagnetic, baryonic and isospin currents on the vorticity comes weighted by the chemical potential $\mu_{5}$ and therefore disappears in the absence of chiral imbalance $\mu_{5} \rightarrow 0$. Despite being erased from the currents, vorticity dependent terms survive in the equilibrium number densities in this limit. Explicit expressions of the outof-equilibrium transport coefficients in the unbroken phase of the theory are also provided.

The article is organized as follows. In section 2 we briefly review the main results of ref. [32] which will be later used in the analysis of hadronic (super)fluids. After this, we study in section 3 a two-flavor hadronic fluid without chiral symmetry breaking and compute its equilibrium partition function, as well as the relevant currents and the energymomentum tensor to leading and first order in the derivative expansion. Section 4 will be devoted to the study of this system in the phase in which chiral symmetry is spontaneously broken, computing the corresponding pion partition function up to first order in derivatives. In section 5 , the covariant currents and constitutive relations of this hadron superfluid will 
be computed, and the emergence of various chiral effects discussed. Finally, we summarize our conclusions in section 6 .

\section{Equilibrium partition functions from differential geometry}

For the reader's convenience and to make our presentation self-contained, in this section we briefly summarize the formalism and main results of ref. [32]. Key expressions will be given in components for their use later on in the paper.

We study a theory of chiral fermions coupled to external gauge fields focusing for the time being on the simplest case of a single gauge potential one-form and its field strength two-form, both taking values on the algebra of the gauge group $\mathcal{G}$

$$
\begin{aligned}
& \mathcal{A}=-i \mathcal{A}_{\mu} d x^{\mu}, \\
& \mathcal{F} \equiv d \mathcal{A}+\mathcal{A}^{2}=-\frac{i}{2} \mathcal{F}_{\mu \nu} d x^{\mu} d x^{\nu} .
\end{aligned}
$$

The main ingredient in the construction of the anomalous effective action in $D=2 n-2$ dimensions, and thus of the equilibrium partition function, is the anomaly polynomial, a $2 n$-form built in terms of $\mathcal{F}$. If the external gauge field $\mathcal{A}$ is coupled to a right-handed fermion, this is given by

$$
\mathcal{P}(\mathcal{F}) \equiv 2 \pi(\operatorname{ch} \mathcal{F})_{2 n}=c_{n} \operatorname{Tr} \mathcal{F}^{n} \quad \text { with } \quad c_{n}=\frac{1}{n !} \frac{i^{n}}{(2 \pi)^{2 n-1}},
$$

where $\operatorname{ch} \mathcal{F}$ is the Chern character (see [36] for definitions and conventions) and its subscript indicates that we only retain the form of degree $2 n$. The global normalization is dictated by the Atiyah-Singer index theorem relating the anomaly with the topological properties of the gauge bundle. The anomalous part of the effective action $\Gamma[\mathcal{A}]_{\mathrm{CS}}$ can be constructed then in terms of the Chern-Simons form $\omega_{2 n-1}^{0}(\mathcal{A}, \mathcal{F})$, defined by

$$
\mathcal{P}(\mathcal{F})=d \omega_{2 n-1}^{0}(\mathcal{A}, \mathcal{F}) \quad \Longrightarrow \quad \Gamma[\mathcal{A}]_{\mathrm{CS}}=c_{n} \int_{\mathcal{M}_{2 n-1}} \omega_{2 n-1}^{0}(\mathcal{A}, \mathcal{F})
$$

In the case of a left-handed fermion, the corresponding expression for the effective action carries an additional global minus sign. For theories with various chiral fermions, the result is obtained by adding the contributions of the different species weighted by the corresponding signs ( + for right- and - for left-handed fermions).

To compute the equilibrium partition function, we define the theory on a generic static background [37] whose metric, upon choosing appropriate coordinates, can be brought to the form

$$
d s^{2} \equiv G_{\mu \nu}(\mathbf{x}) d x^{\mu} d x^{\nu}=-e^{2 \sigma(\mathbf{x})}\left[d t+a_{i}(\mathbf{x}) d x^{i}\right]^{2}+g_{i j}(\mathbf{x}) d x^{i} d x^{j} .
$$

Written like this, the metric has manifest timelike Killing vector given by $\partial_{t}$. We also take all other tensor fields in the theory to have vanishing Lie derivative with respect to this Killing field, which in the coordinates chosen simply means that they are time-independent. 
The form of the line element (2.4) is preserved by Kaluza-Klein (KK) transformations, defined by position-dependent shifts generated by the vector field $\zeta(\mathbf{x})=\phi(\mathbf{x}) \partial_{t}$ together with an appropriate redefinition of the metric function $a_{i}(\mathbf{x})$, namely

$$
\begin{aligned}
(t, \mathbf{x}) & \longrightarrow(t+\phi, \mathbf{x}), \\
a_{i} & \longrightarrow a_{i}-\partial_{i} \phi .
\end{aligned}
$$

The diffeomorphism generated by $\zeta(\mathbf{x})$ induces the following change on the components of gauge field one-forms defined on this spacetime

$$
\begin{aligned}
& \mathcal{A}_{0} \longrightarrow \mathcal{A}_{0}, \\
& \mathcal{A}_{i} \longrightarrow \mathcal{A}_{i}-\mathcal{A}_{0} \partial_{i} \phi .
\end{aligned}
$$

Associated with this metric, we can introduce the properly normalized four-velocity of comoving observers in the static metric (2.4)

$$
u^{\mu}=e^{-\sigma}(1, \mathbf{0}) \quad \Longrightarrow \quad u_{\mu}=-e^{\sigma}\left(1, a_{i}\right) .
$$

This defines a congruence of timelike geodesics foliating the spacetime by spatial hypersurfaces normal to $u^{\mu}$, whose induced metric is given by

$$
h_{\mu \nu}=G_{\mu \nu}+u_{\mu} u_{\nu} \quad \Longrightarrow \quad h_{i j}=g_{i j},
$$

with $h_{00}=h_{0 i}=0$.

Tensor fields in this geometry can be decomposed into KK-invariant combinations by splitting them into longitudinal and transverse components with respect to the vector $u^{\mu}$. To do this, we introduce the projector

$$
P_{\nu}^{\mu} \equiv h_{\nu}^{\mu}=\delta_{\nu}^{\mu}+u^{\mu} u_{\nu}=\left(\begin{array}{rr}
0 & -a_{i} \\
0 & \delta_{j}^{i}
\end{array}\right),
$$

and contract indices with the identity written in the form $\delta^{\mu}{ }_{\nu}=-u^{\mu} u_{\nu}+P_{\nu}^{\mu}$. In the case of a gauge field, the result is

$$
\mathcal{A}_{\mu}=-\left(\mathcal{A}_{\nu} u^{\nu}\right) u_{\mu}+\mathcal{A}_{\nu} P_{\mu}^{\nu}
$$

Scalar quantities are, by definition, invariant under KK transformations and so is the combination appearing in the first term

$$
\mathcal{A}_{\mu} u^{\mu}=e^{-\sigma} \mathcal{A}_{0} \equiv e^{-\sigma} A_{0} .
$$

For the transverse components, using the explicit form of the projector, we find

$$
\mathcal{A}_{\nu} P^{\nu}{ }_{\mu}=\left(0, \mathcal{A}_{i}-\mathcal{A}_{0} a_{i}\right) \equiv\left(0, A_{i}\right) .
$$

This pulled-back one-form, having a vanishing time component, is automatically KKinvariant as can be checked from eq. (2.6). Thus, gauge field one-form in eq. (2.1) can be written in terms of KK-invariant components defined by

$$
\begin{aligned}
& A_{0} \equiv e^{\sigma} u^{\mu} \mathcal{A}_{\mu}=\mathcal{A}_{0}, \\
& A_{i} \equiv \mathcal{A}_{\mu} P_{i}^{\mu}=\mathcal{A}_{i}-\mathcal{A}_{0} a_{i} .
\end{aligned}
$$


From here we retrieve the decomposition used in [32]

$$
\mathcal{A}=-i \mathcal{A}_{\nu}\left(-u^{\nu} u_{\mu}+P_{\mu}^{\nu}\right) d x^{\mu} \equiv-i \mathcal{A}_{0} \theta(\mathbf{x})+\boldsymbol{A}=-i A_{0} \theta-i A_{i} d x^{i},
$$

where we have defined the one-form $\theta \equiv-e^{-\sigma} u_{\mu} d x^{\mu}$. Together with $d x^{i}$, it makes up a KK-invariant cotangent basis. ${ }^{1}$ The relations (2.13) can be inverted to write

$$
\mathcal{A}_{\mu}=-e^{-\sigma} A_{0} u_{\mu}+A_{i} \delta_{\mu}^{i} \text {. }
$$

Similar decompositions can be written also for a vector field $\mathcal{W}^{\mu} \partial_{\mu}$ in terms of KK-invariant components $\left(W^{0}, W^{i}\right)$, by expanding it in the KK-invariant tangent basis $\left\{u^{\mu} \partial_{\mu}, P_{i}^{\mu} \partial_{\mu}\right\}=$ $\left\{e^{\sigma} \partial_{0}, \partial_{i}-a_{i} \partial_{0}\right\}$

$$
\begin{aligned}
\mathcal{W}^{\mu} \partial_{\mu} & =-\left(\mathcal{W}^{\nu} u_{\nu}\right) u^{\mu} \partial_{\mu}+P_{i}^{\mu} \mathcal{W}^{i} \partial_{\mu} \\
& =e^{\sigma} W^{0} \partial_{0}+W^{i}\left(\partial_{i}-a_{i} \partial_{0}\right)=\left(e^{\sigma} W^{0}-W^{i} a_{i}\right) \partial_{0}+W^{i} \partial_{i},
\end{aligned}
$$

so we have

$$
\mathcal{W}^{\mu}=\left(e^{\sigma} W^{0}-W^{i} a_{i}\right) u^{\mu}+W^{i} \delta_{i}^{\mu} .
$$

The notation used in this paper is a slight modification of the one introduced in ref. [32]. We reserve calligraphic script $\mathcal{A}, \mathcal{F}, \ldots$ to denote the $K K$-variant components. Roman script $A, F, \ldots$ indicates the corresponding $K K$-invariant fields. From eq. (2.6), we notice that the time component of the gauge field $\mathcal{A}_{0}$ is KK invariant, so we have $\mathcal{A}_{0}=A_{0}$. However, unlike in ref. [32], here we only use Hermitian components for the diverse adjoint fields, as can be seen from the presence of the $-i$ factor in eq. (2.1).

To construct the equilibrium partition function at temperature $T_{0} \equiv \beta^{-1}$, we use the imaginary time formalism setting in (2.3) a manifold $\mathcal{M}_{2 n-1}$ with topology $S^{1} \times D_{2 n-2}$, where the second factor is a $(2 n-2)$-dimensional ball and the thermal cycle $S^{1}$ has length $\beta$. Since all fields are time-independent, the integration over $d x^{0}$ is trivially implemented by replacing

$$
i \int_{S^{1} \times D_{2 n-2}} d t \longrightarrow \frac{1}{T_{0}} \int_{D_{2 n-2}} .
$$

Using this prescription, one finds that the equilibrium partition function has the following structure

$$
i \Gamma\left[\mathcal{A}_{0}, \boldsymbol{A}\right]_{\mathrm{CS}}=W\left[\mathcal{A}_{0}, \boldsymbol{A}\right]_{\mathrm{inv}}+W\left[\mathcal{A}_{0}, \boldsymbol{A}\right]_{\mathrm{anom}},
$$

where the first piece is gauge invariant and the anomaly is carried by the second term. This decomposition was found in [24], while in [32] we provided closed expressions for both terms using generalized transgression [38]. The first, gauge invariant piece can be written as the following integral over the bulk manifold $D_{2 n-2}$

$$
W\left[\mathcal{A}_{0}, \boldsymbol{A}\right]_{\mathrm{inv}}=-\frac{i n c_{n}}{T_{0}} \int_{D_{2 n-2}} \int_{0}^{1} d t \operatorname{Tr}\left[\mathcal{A}_{0}\left(\boldsymbol{F}-i t \mathcal{A}_{0} d a\right)^{n-1}\right],
$$

\footnotetext{
${ }^{1}$ The KK-invariant gauge one-form $A_{i} d x^{i}$ corresponds to the hatted connection used in ref. [24].
} 
where we have introduced the KK gauge field one-form $a \equiv a_{i} d x^{i}$. Being a higherdimensional integral, this term is nonlocal in physical space. The anomalous part of the partition function, on the other hand, takes the form

$$
W\left[\mathcal{A}_{0}, \boldsymbol{A}\right]_{\mathrm{anom}}=-\left.\frac{i c_{n}}{T_{0}} \int_{S^{2 n-3}} \int_{0}^{1} d t \mathcal{A}_{0} \frac{\delta}{\delta \mathcal{F}} \omega_{2 n-1}^{0}(\mathcal{A}, \mathcal{F})\right|_{\substack{\mathcal{A} \rightarrow \boldsymbol{A} \\ \mathcal{F} \rightarrow \boldsymbol{F}-i t \mathcal{A}_{0} d a}}
$$

where $S^{2 n-3} \equiv \partial D_{2 n-2}$ is the physical space. This contribution to the effective action not only carries the anomaly, but is actually local.

It should be stressed at this point that, despite its gauge invariance, the nonlocal piece in (2.19) is crucial for the computation of the covariant currents and the anomalous contribution to the energy-momentum tensor. Indeed, covariant and consistent currents are then derived from the variation of the invariant and anomalous pieces of the partition function under $\delta_{B} \mathcal{A}=\mathcal{B}_{0} \theta+\boldsymbol{B}$

$$
\begin{aligned}
\delta_{B} W\left[\mathcal{A}_{0}, \boldsymbol{A}\right]_{\mathrm{inv}} & =\int_{S^{2 n-3}} \operatorname{Tr}\left(-i \mathcal{B}_{0} \mathcal{J}_{0, \mathrm{cov}}+\boldsymbol{B} \boldsymbol{J}_{\text {cov }}\right)+\text { bulk terms } \\
\delta_{B} W\left[\mathcal{A}_{0}, \boldsymbol{A}\right]_{\text {anom }} & =\int_{S^{2 n-3}} \operatorname{Tr}\left(-i \mathcal{B}_{0} \mathcal{J}_{0, \mathrm{cons}}+\boldsymbol{B} \boldsymbol{J}_{\text {cons }}\right) .
\end{aligned}
$$

In fact, the local character of the anomalous part of the effective action in (2.19) can be derived on more general grounds. To fix ideas, let us consider the simplest case of a left- and a right-handed fermion coupled to external $\mathrm{U}(1)$ vector $\mathcal{V}_{\mu}=\left(\mathcal{V}_{0}, \mathcal{V}\right)$ and axialvector $\mathcal{A}_{\mu}=\left(\mathcal{A}_{0}, \mathcal{A}\right)$ external gauge fields in flat four-dimensional spacetime. The nonlocal anomalous effective action for these fields can be written as [39]

$$
S[\mathcal{V}, \mathcal{A}]_{\mathrm{eff}}=-\frac{e^{2}}{16 \pi^{2}} \int d^{4} x \int d^{4} y\left(\epsilon^{\mu \nu \alpha \beta} \mathcal{V}_{\mu \nu} \mathcal{V}_{\alpha \beta}\right)_{x}\left(\frac{1}{\square}\right)_{x y}\left(\partial_{\sigma} \mathcal{A}^{\sigma}\right)_{y}
$$

where $\mathcal{V}_{\mu \nu}=\partial_{\mu} \mathcal{V}_{\nu}-\partial_{\nu} \mathcal{V}_{\mu}$ is the field strength associated with the Abelian vector gauge field. In the static case where all fields are time-independent, we can implement dimensional reduction and the effective action simplifies to

$$
\begin{aligned}
S[\mathcal{V}, \mathcal{A}]_{\mathrm{eff}} & =\frac{i e^{2}}{4 \pi^{2} T_{0}} \int d^{3} \mathbf{x} \int d^{3} \mathbf{y}\left(\epsilon^{i j k} \mathcal{V}_{i j} \mathcal{V}_{0 k}\right)_{\mathbf{x}}\left(\frac{1}{\boldsymbol{\nabla}^{2}}\right)_{\mathbf{x y}}(\boldsymbol{\nabla} \cdot \mathcal{A})_{\mathbf{y}} \\
& =-\frac{i e^{2}}{4 \pi^{2} T_{0}} \int d^{3} \mathbf{x} \int d^{3} \mathbf{y}\left(\boldsymbol{\nabla} \mathcal{V}_{0} \cdot \mathcal{B}\right)_{\mathbf{x}}\left(\frac{1}{\boldsymbol{\nabla}^{2}}\right)_{\mathbf{x y}}(\boldsymbol{\nabla} \cdot \mathcal{A})_{\mathbf{y}}
\end{aligned}
$$

where we have identified the electric and magnetic fields associated to the vector gauge connection, $\mathcal{E}=-\boldsymbol{\nabla} \mathcal{V}_{0}$ and $\mathcal{B}=\boldsymbol{\nabla} \times \mathcal{V}$. Taking into account that $\boldsymbol{\nabla} \cdot \mathcal{B}=0$ and integrating by parts twice, we find

$$
\begin{aligned}
S[\mathcal{V}, \mathcal{A}]_{\mathrm{eff}} & =-\frac{i e^{2}}{4 \pi^{2} T_{0}} \int d^{3} \mathbf{x} \int d^{3} \mathbf{y}\left(\mathcal{V}_{0} \mathcal{B}\right)_{\mathbf{x}}\left[\boldsymbol{\nabla}_{\mathbf{y}} \cdot \nabla_{\mathbf{x}}\left(\frac{1}{\nabla^{2}}\right)_{\mathbf{x y}}\right] \mathcal{A}_{\mathbf{y}} \\
& =-\frac{i e^{2}}{4 \pi^{2} T_{0}} \int d^{3} \mathbf{x}\left(\mathcal{V}_{0} \mathcal{B} \cdot \mathcal{A}\right)_{\mathbf{x}}
\end{aligned}
$$


which is indeed a local functional in three dimensions. This expression is invariant under vector gauge transformations (notice that $\mathcal{V}_{0}$ does not change when the gauge parameter is time-independent), whereas its variation under axial-vector gauge transformations $\mathcal{A} \rightarrow$ $\mathcal{A}+\nabla \eta$ reproduces the axial anomaly

$$
\delta_{\eta} S[\mathcal{V}, \mathcal{A}]_{\mathrm{eff}}=-\frac{i e^{2}}{4 \pi^{2} T_{0}} \int d^{3} \mathbf{x} \eta \mathcal{E} \cdot \mathcal{B}
$$

In ref. [32] a general prescription was given allowing the construction of the equilibrium partition function of generic theories, as well their consistent and covariant currents. Concerning the energy-momentum tensor, the Chern-Simons effective action (2.3) is a topological invariant and therefore independent of the metric defined on the manifold $\mathcal{M}_{2 n-1}$. Naively, it would seem then that the anomaly does not induce any terms in the energymomentum tensor. However, this is not so. As a result of the dimensional reduction (2.18) based on the KK-invariant decomposition (2.14), the partition function acquires an explicit dependence on the metric function $a_{i}(\mathbf{x})$, which brings about a nonzero anomalous contribution to the $T_{0}{ }^{i}$ component of the energy-momentum tensor.

The Bardeen anomaly. From now on we focus our analysis on the case of the Bardeen anomaly in $D=4(n=3)$, where the corresponding equilibrium partition function depends on non-Abelian vector $\mathcal{V}=-i \mathcal{V}_{\mu} d x^{\mu}$ and axial-vector $\mathcal{A}=-i \mathcal{A}_{\mu} d x^{\mu}$ external gauge fields. The appropriate Chern-Simons form preserving the invariance under vector gauge transformations takes the form (see [32] for details)

$$
\widetilde{\omega}_{5}^{0}\left(\mathcal{A}, \mathcal{F}_{V}, \mathcal{F}_{A}\right)=6 \operatorname{Tr}\left(\mathcal{A} \mathcal{F}_{V}^{2}+\frac{1}{3} \mathcal{A F}_{A}^{2}-\frac{4}{3} \mathcal{A}^{3} \mathcal{F}_{V}+\frac{8}{15} \mathcal{A}^{5}\right)
$$

where the field strengths are given in components by

$$
\begin{aligned}
\mathcal{F}_{V} & =-\frac{i}{2} \mathcal{V}_{\mu \nu} d x^{\mu} d x^{\nu} \equiv-\frac{i}{2}\left(\partial_{\mu} \mathcal{V}_{\nu}-\partial_{\nu} \mathcal{V}_{\mu}-i\left[\mathcal{V}_{\mu}, \mathcal{V}_{\nu}\right]-i\left[\mathcal{A}_{\mu}, \mathcal{A}_{\nu}\right]\right) d x^{\mu} d x^{\nu} \\
\mathcal{F}_{A} & =-\frac{i}{2} \mathcal{A}_{\mu \nu} d x^{\mu} d x^{\nu} \equiv-\frac{i}{2}\left(\partial_{\mu} \mathcal{A}_{\nu}-\partial_{\nu} \mathcal{A}_{\mu}-i\left[\mathcal{V}_{\mu}, \mathcal{A}_{\nu}\right]-i\left[\mathcal{A}_{\mu}, \mathcal{V}_{\nu}\right]\right) d x^{\mu} d x^{\nu}
\end{aligned}
$$

After implementing dimensional reduction and writing everything in terms of the corresponding KK-invariant fields $\left(\mathcal{V}_{0}, V_{i}\right)$ and $\left(\mathcal{A}_{0}, A_{i}\right)$ [cf. (2.14)], we find that the invariant and anomalous parts of the anomalous functional can be computed by the likes of eqs. (2.20) and (2.21) (details and the explicit expressions can be found in ref. [32]). The expectation values of the two-forms dual to the spatial vector and axial-vector covariant currents can be obtained by functional differentiation from the invariant nonlocal part of the partition function according to $[24,32]$

$$
\begin{aligned}
& \left\langle\boldsymbol{J}_{V}\right\rangle_{\mathrm{cov}} \equiv\left\langle\star \boldsymbol{j}_{V}\right\rangle_{\mathrm{cov}}=T_{0} \frac{\delta}{\delta \boldsymbol{F}_{V}} W\left[\mathcal{V}_{0}, \mathcal{A}_{0}, \boldsymbol{F}_{V}, \boldsymbol{F}_{A}\right]_{\mathrm{inv}}, \\
& \left\langle\boldsymbol{J}_{A}\right\rangle_{\mathrm{cov}} \equiv\left\langle\star \boldsymbol{j}_{A}\right\rangle_{\mathrm{cov}}=T_{0} \frac{\delta}{\delta \boldsymbol{F}_{A}} W\left[\mathcal{V}_{0}, \mathcal{A}_{0}, \boldsymbol{F}_{V}, \boldsymbol{F}_{A}\right]_{\mathrm{inv}},
\end{aligned}
$$


where the star represents the Hodge dual on the spatial $S^{3}$. An explicit expression of these currents has been given in ref. [32]. Writing

$$
\boldsymbol{j}=-i \mathcal{J}_{k} d x^{k}
$$

and expressing the KK-invariant vector and axial-vector gauge forms in terms of their components

$$
\begin{array}{lll}
\boldsymbol{V} & =-i V_{i} d x^{i}, \\
\boldsymbol{A} & =-i A_{i} d x^{i},
\end{array} \quad \Longrightarrow \quad \begin{aligned}
& \boldsymbol{F}_{V}=-\frac{i}{2} V_{i j} d x^{i} d x^{j}, \\
& \boldsymbol{F}_{A}=-\frac{i}{2} A_{i j} d x^{i} d x^{j},
\end{aligned}
$$

we arrive at the expressions

$$
\begin{aligned}
& \left\langle\mathcal{J}_{a V}^{i}\right\rangle_{\mathrm{cov}}=\frac{N_{c}}{8 \pi^{2}} e^{-\sigma} \epsilon^{i k \ell} \operatorname{Tr}\left\{t_{a}\left[V_{0} A_{k \ell}+A_{k \ell} V_{0}+A_{0} V_{k \ell}+V_{k \ell} A_{0}+2\left(V_{0} A_{0}+A_{0} V_{0}\right) \partial_{k} a_{\ell}\right]\right\}, \\
& \left\langle\mathcal{J}_{a A}^{i}\right\rangle_{\mathrm{cov}}=\frac{N_{c}}{8 \pi^{2}} e^{-\sigma} \epsilon^{i k \ell} \operatorname{Tr}\left\{t_{a}\left[V_{0} V_{k \ell}+V_{k \ell} V_{0}+A_{0} A_{k \ell}+A_{k \ell} A_{0}+2\left(V_{0}^{2}+A_{0}^{2}\right) \partial_{k} a_{\ell}\right]\right\},
\end{aligned}
$$

where, for later use, we have assumed that chiral fermions coupling to the external fields come in $N_{c}$ different colors. To keep the notation homogeneous, we used $\mathcal{V}_{0}=V_{0}$ and $\mathcal{A}_{0}=A_{0}$. In addition to this, the covariant time components are zero for both the vector and axial-vector covariant currents [24, 32]

$$
\left\langle\mathcal{J}_{a 0 V}\right\rangle_{\mathrm{cov}}=\left\langle\mathcal{J}_{a 0 A}\right\rangle_{\mathrm{cov}}=0 .
$$

Raising the index using the static metric (2.4), we find

$$
\begin{aligned}
& \left\langle\mathcal{J}_{a V}^{0}\right\rangle_{\mathrm{cov}}=-a_{i}\left\langle\mathcal{J}_{a V}^{i}\right\rangle_{\mathrm{cov}}, \\
& \left\langle\mathcal{J}_{a A}^{0}\right\rangle_{\mathrm{cov}}=-a_{i}\left\langle\mathcal{J}_{a A}^{i}\right\rangle_{\mathrm{cov}},
\end{aligned}
$$

where the right-hand side of these expressions can be read off from eq. (2.32) and spatial indices are raised and lowered using the three-dimensional transverse metric $g_{i j}$. The flavor components of the current are defined by

$$
\mathcal{J}_{a}^{\mu} \equiv \operatorname{Tr}\left(t_{a} \mathcal{J}^{\mu}\right)
$$

with $t_{a}$ the gauge group generators. Identities (2.34) can be written in a Lorentz-covariant way by stating that the covariant current is transverse to the four-velocity, namely

$$
u_{\mu}\left\langle\mathcal{J}_{a V}^{\mu}\right\rangle_{\mathrm{cov}}=u_{\mu}\left\langle\mathcal{J}_{a A}^{\mu}\right\rangle_{\mathrm{cov}}=0 .
$$

As for the anomaly-induced component of the energy-momentum tensor, its dual twoform can be also obtained by taking functional derivatives of the invariant part of the effective action [32]

$$
\boldsymbol{T}\left(\mathcal{V}_{0}, \boldsymbol{V}, \mathcal{A}_{0}, \boldsymbol{A}\right)=T_{0}\left[\frac{\delta}{\delta(d a)}+i \mathcal{V}_{0} \frac{\delta}{\delta \boldsymbol{F}_{V}}+i \mathcal{A}_{0} \frac{\delta}{\delta \boldsymbol{F}_{A}}\right] W\left[\mathcal{V}_{0}, \mathcal{A}_{0}, \boldsymbol{F}_{V}, \boldsymbol{F}_{A}\right]_{\mathrm{inv}}
$$


Taking the Hodge dual and writing the equation in components, we find the explicit expression

$$
\begin{aligned}
T_{0}{ }^{i}\left(V_{0}, A_{0}, V_{i}, A_{i}\right)=-\frac{N_{c}}{8 \pi^{2}} e^{-\sigma} \epsilon^{i j k} \operatorname{Tr}\left[V_{j k}\left(V_{0} A_{0}+A_{0} V_{0}\right)\right. & +A_{j k}\left(V_{0}^{2}+A_{0}^{2}\right) \\
& \left.+\frac{2}{3} \partial_{j} a_{k}\left(A_{0}^{3}+3 A_{0} V_{0}^{2}\right)\right] .
\end{aligned}
$$

Nambu-Goldstone bosons. The differential geometry approach to the computation of covariant currents is specially useful when applied to systems with spontaneous symmetry breaking. In this case, the dynamics of NG bosons is largely determined by the anomaly through the following prescription for the WZW effective action [1]

$$
\Gamma[A, U]_{\mathrm{WZW}}=\Gamma[\mathcal{A}]_{\mathrm{CS}}-\Gamma\left[\mathcal{A}^{U}\right]_{\mathrm{CS}},
$$

where $\mathcal{A}^{U}=U^{-1} \mathcal{A} U+U^{-1} d U$ is the gauge connection transformed by $U$. The corresponding equilibrium partition function is computed implementing the dimensional reduction to give [32]

$$
\begin{aligned}
W\left[\mathcal{A}_{0}, \boldsymbol{A}, U\right]_{\mathrm{WZW}} & =i \Gamma\left[\mathcal{A}_{0}, \boldsymbol{A}\right]_{\mathrm{CS}}-i \Gamma\left[\mathcal{A}_{0}^{U}, \boldsymbol{A}^{U}\right]_{\mathrm{CS}} \\
& =W\left[\mathcal{A}_{0}, \boldsymbol{A}\right]_{\mathrm{anom}}-W\left[\mathcal{A}_{0}, \boldsymbol{A}+d U U^{-1}\right]_{\mathrm{anom}}
\end{aligned}
$$

Here, the gauge invariant nonlocal piece of the partition function in eq. (2.19) cancels between the two terms in the first line. Thus, the partition function for NG bosons can be computed using the prescription (2.21). Again, we focus our analysis to the case of the Bardeen anomaly, where chiral fermions couple to vector and axial-vector external gauge fields. If the gauge group $\mathcal{G} \times \mathcal{G}$ breaks down to its vector subroup, $\mathcal{G} \times \mathcal{G} \rightarrow \mathcal{G}$, the resulting WZW partition function has the structure

$$
W\left[\mathcal{V}_{0}, \mathcal{A}_{0}, \boldsymbol{V}, \boldsymbol{A}, U\right]_{\mathrm{WZW}}=W\left[\mathcal{V}_{0}, \mathcal{A}_{0}, \boldsymbol{V}, \boldsymbol{A}\right]_{\mathrm{anom}}-W\left[\mathcal{V}_{0}^{U}, \mathcal{A}_{0}^{U}, \boldsymbol{V}^{U}, \boldsymbol{A}^{U}\right]_{\mathrm{anom}},
$$

where the transformed dimensionally-reduced gauge fields are given by

$$
\begin{aligned}
& \mathcal{V}_{0}^{U}=\frac{1}{2}\left(\mathcal{V}_{0}+\mathcal{A}_{0}\right)+\frac{1}{2} U^{-1}\left(\mathcal{V}_{0}-\mathcal{A}_{0}\right) U, \\
& \mathcal{A}_{0}^{U}=\frac{1}{2}\left(\mathcal{V}_{0}+\mathcal{A}_{0}\right)-\frac{1}{2} U^{-1}\left(\mathcal{V}_{0}-\mathcal{A}_{0}\right) U,
\end{aligned}
$$

together with

$$
\begin{aligned}
& \boldsymbol{V}^{U}=\frac{1}{2}(\boldsymbol{V}+\boldsymbol{A})+\frac{1}{2} U^{-1}(\boldsymbol{V}-\boldsymbol{A}) U+\frac{1}{2} U^{-1} d U \\
& \boldsymbol{A}^{U}=\frac{1}{2}(\boldsymbol{V}+\boldsymbol{A})-\frac{1}{2} U^{-1}(\boldsymbol{V}-\boldsymbol{A}) U-\frac{1}{2} U^{-1} d U .
\end{aligned}
$$

One of the main results of ref. [32] is that the covariant current for systems with spontaneous symmetry breaking can be computed without resorting to the WZW effective action, which usually contains a large number of terms. Instead, they can be obtained from 
the (Hodge dual) BZ current $\mathcal{J}(\mathcal{A})_{\mathrm{BZ}}$ of the unbroken theory. For the Bardeen anomaly, the vector and axial-vector parts of the covariant currents can be obtained from the identity

$$
c_{n} \ell \widetilde{\omega}_{2 n-1}^{0}(\mathcal{V}, \mathcal{A})=-\operatorname{Tr}\left(B_{V} \mathcal{J}_{\mathrm{BZ}}^{V}+B_{A} \mathcal{J}_{\mathrm{BZ}}^{A}\right),
$$

where $\widetilde{\omega}_{2 n-1}^{0}(\mathcal{V}, \mathcal{A})$ is the Chern-Simons form preserving vector gauge transformations, while the operator $\ell$ is defined by its action over the gauge field and field strength forms [38]

$$
\ell \mathcal{V}=\ell \mathcal{A}=0, \quad \ell \mathcal{F}_{V}=B_{V}, \quad \ell \mathcal{F}_{A}=B_{A}
$$

In four-dimensions, the relevant Chern-Simons form $\widetilde{\omega}_{5}^{0}(\mathcal{V}, \mathcal{A})$ is given in eq. (2.27). After a short calculation, one arrives at the following expressions for the BZ currents in terms of KK-variant vector and axial-vector gauge fields and their fields strengths

$$
\begin{aligned}
& \left\langle\mathcal{J}_{a V}^{\mu}\right\rangle_{\mathrm{BZ}}=-\frac{N_{c}}{8 \pi^{2}} \epsilon^{\mu \nu \alpha \beta} \operatorname{Tr}\left[t_{a}\left(\mathcal{V}_{\nu \alpha} \mathcal{A}_{\beta}+\mathcal{A}_{\nu} \mathcal{V}_{\alpha \beta}+\frac{8 i}{3} \mathcal{A}_{\nu} \mathcal{A}_{\alpha} \mathcal{A}_{\beta}\right)\right], \\
& \left\langle\mathcal{J}_{a A}^{\mu}\right\rangle_{\mathrm{BZ}}=-\frac{N_{c}}{24 \pi^{2}} \epsilon^{\mu \nu \alpha \beta} \operatorname{Tr}\left[t_{a}\left(\mathcal{A}_{\nu \alpha} \mathcal{A}_{\beta}+\mathcal{A}_{\nu} \mathcal{A}_{\alpha \beta}\right)\right],
\end{aligned}
$$

where $t_{a}$ are the generators of the gauge group $\mathcal{G}$.

Once the BZ currents are known, the right- and left-handed components of the (dual) covariant current in the spontaneously broken symmetry phase is given by the simple relation $[32]$

$$
\begin{aligned}
\mathcal{J}_{\mu}^{R}\left(\mathcal{A}_{R}, \mathcal{A}_{L}, U\right)_{\mathrm{cov}} & =\mathcal{J}_{\mu}^{R}\left(\mathcal{A}_{R}, \mathcal{A}_{L}^{U}\right)_{\mathrm{BZ}}, \\
\mathcal{J}_{\mu}^{L}\left(\mathcal{A}_{R}, \mathcal{A}_{L}, U\right)_{\mathrm{cov}} & =U \mathcal{J}_{\mu}^{L}\left(\mathcal{A}_{R}, \mathcal{A}_{L}^{U}\right)_{\mathrm{BZ}} U^{-1},
\end{aligned}
$$

where the $\mathcal{A}_{R}$ and $\mathcal{A}_{L}$ gauge fields are defined in terms of $\mathcal{V}_{\mu}$ and $\mathcal{A}_{\mu}$ respectively by

$$
\begin{aligned}
& \mathcal{A}_{R}=\mathcal{V}+\mathcal{A}, \\
& \mathcal{A}_{L}=\mathcal{V}-\mathcal{A},
\end{aligned}
$$

while the corresponding components of the BZ left- and right-handed one-form currents are

$$
\begin{aligned}
\mathcal{J}^{R}(\mathcal{V}, \mathcal{A})_{\mathrm{BZ}} & =\frac{1}{2}\left[\mathcal{J}^{V}(\mathcal{V}, \mathcal{A})_{\mathrm{BZ}}+\mathcal{J}^{A}(\mathcal{V}, \mathcal{A})_{\mathrm{BZ}}\right] \\
\mathcal{J}^{L}(\mathcal{V}, \mathcal{A})_{\mathrm{BZ}} & =\frac{1}{2}\left[\mathcal{J}^{V}(\mathcal{V}, \mathcal{A})_{\mathrm{BZ}}-\mathcal{J}^{A}(\mathcal{V}, \mathcal{A})_{\mathrm{BZ}}\right] .
\end{aligned}
$$

The expressions for $\mathcal{J}_{\text {cov }}^{R}$ and $\mathcal{J}_{\text {cov }}^{L}$ have been computed in [32] using (2.46). Here we write them in flavor components

$$
\begin{aligned}
& \mathcal{J}_{a}^{R}\left(\mathcal{A}_{R}, \mathcal{A}_{L}, U\right)_{\mathrm{cov}}=-\frac{i}{24 \pi^{2}} \operatorname{Tr}\left\{t_{a}\right. {\left[\left(U^{-1} \mathcal{A}_{L} U-\mathcal{A}_{R}+U_{R}\right)\left(\mathcal{F}_{R}+\frac{1}{2} U^{-1} \mathcal{F}_{L} U\right)\right.} \\
&+\left(\mathcal{F}_{R}+\frac{1}{2} U^{-1} \mathcal{F}_{L} U\right)\left(U^{-1} \mathcal{A}_{L} U-\mathcal{A}_{R}+U_{R}\right) \\
&-\left.\left.\frac{1}{2}\left(U^{-1} \mathcal{A}_{L} U-\mathcal{A}_{R}+U_{R}\right)^{3}\right]\right\}, \\
& \mathcal{J}_{a}^{L}\left(\mathcal{A}_{R}, \mathcal{A}_{L}, U\right)_{\mathrm{cov}}=-\frac{i}{24 \pi^{2}} \operatorname{Tr}\left\{t _ { a } \left[\left(\mathcal{A}_{L}-U \mathcal{A}_{R} U^{-1}+U_{L}\right)\left(\mathcal{F}_{L}+\frac{1}{2} U \mathcal{F}_{R} U^{-1}\right)\right.\right. \\
&+\left(\mathcal{F}_{L}+\frac{1}{2} U \mathcal{F}_{R} U^{-1}\right)\left(\mathcal{A}_{L}-U \mathcal{A}_{R} U^{-1}+U_{L}\right) \\
&\left.\left.-\frac{1}{2}\left(\mathcal{A}_{L}-U \mathcal{A}_{R} U^{-1}+U_{L}\right)^{3}\right]\right\},
\end{aligned}
$$


where the notation

$$
U_{R}=U^{-1} d U, \quad U_{L}=d U U^{-1}
$$

has been used. Once these left- and right-handed currents have been evaluated, the vector and axial-vector covariant dual currents are obtained by

$$
\begin{aligned}
& \mathcal{J}_{a}^{V}(\mathcal{V}, \mathcal{A}, U)_{\mathrm{cov}}=\mathcal{J}_{a}^{R}(\mathcal{V}+\mathcal{A}, \mathcal{V}-\mathcal{A}, U)_{\mathrm{cov}}+\mathcal{J}_{a}^{L}(\mathcal{V}+\mathcal{A}, \mathcal{V}-\mathcal{A}, U)_{\mathrm{cov}} \\
& \mathcal{J}_{a}^{A}(\mathcal{V}, \mathcal{A}, U)_{\mathrm{cov}}=\mathcal{J}_{a}^{R}(\mathcal{V}+\mathcal{A}, \mathcal{V}-\mathcal{A}, U)_{\mathrm{cov}}-\mathcal{J}_{a}^{L}(\mathcal{V}+\mathcal{A}, \mathcal{V}-\mathcal{A}, U)_{\mathrm{cov}}
\end{aligned}
$$

\section{Two-flavor QCD fluid with unbroken chiral symmetry}

We apply the formalism reviewed above first to the computation of the covariant currents and the energy-momentum tensor of a QCD fluid with two flavors on the static geometry (2.4). We work in the chiral limit, where the global symmetry group is $\mathrm{U}(2)_{L} \times \mathrm{U}(2)_{R}$. Since the gauge group is non-Abelian, the maximal number of chemical potentials that can be consistently introduced equals the dimension of the Cartan subalgebra [40, 41]. In our case, the $\mathrm{U}(2)$ factors are generated by

$$
t_{0}=\frac{1}{2} \mathbb{1}, \quad t_{i}=\frac{1}{2} \sigma_{i}
$$

with $\sigma_{i}$ the Pauli matrices. Accordingly, we take background fields lying on the combination of the generators $t_{0}$ and $t_{3}$ and define the KK-invariant background

$$
\begin{aligned}
V_{0}(\mathbf{x}) & =V_{00}(\mathbf{x}) t_{0}+V_{30}(\mathbf{x}) t_{3} \\
V_{i}(\mathbf{x}) & =V_{0 i}(\mathbf{x}) t_{0}+V_{3 i}(\mathbf{x}) t_{3} \\
A_{0}(\mathbf{x}) & =A_{00}(\mathbf{x}) t_{0} \\
A_{i}(\mathbf{x}) & =0
\end{aligned}
$$

Furthermore, we take a nonzero constant value for $A_{00}$, which is related to the axial chemical potential $\mu_{5}$ controlling chiral imbalance by ${ }^{2}$

$$
\mu_{5}=e^{-\sigma} A_{00} .
$$

The other two chemical potentials $\mu_{0}$ and $\mu_{3}$ are associated with the time-components of the vector gauge fields according to

$$
\begin{aligned}
& \mu_{0}=e^{-\sigma} V_{00}, \\
& \mu_{3}=e^{-\sigma} V_{30},
\end{aligned}
$$

and respectively control baryon number and isospin imbalance. By choosing $A_{00}$ to be constant, we are focusing on corrections to the covariant currents and energy-momentum

\footnotetext{
${ }^{2}$ This definition of the axial chemical potential differ by a factor of 2 from the one used in [24, 32], where this quantity was introduced through the identity $\mathcal{A}_{\mu}=A_{i} \delta_{\mu}^{i}-\mu_{5} u_{\mu} \mathbb{1}$. To compare with the results of these references, one should replace $\mu_{5} \rightarrow 2 \mu_{5}$ in our expressions.
} 
tensor that contain a single derivative of the vector background fields. In addition, as in $[42,43]$, we have not included terms in $A_{0}$ proportional to $t_{3}$, which would contribute to the axial-vector current.

Using eq. (2.15), the gauge fields can be written in a formally Lorentz covariant form in terms of the chemical potentials and the four-velocity. For $\mathcal{A}_{0 \mu}$, since the spatial components are zero, the gauge field itself is proportional to the four-velocity vector

$$
\mathcal{A}_{0 \mu}=-e^{-\sigma} A_{00} u_{\mu}=-\mu_{5} u_{\mu} .
$$

The associated field strength can then be written as

$$
\mathcal{A}_{0 \mu \nu}=-\mu_{5}\left(\partial_{\mu} u_{\nu}-\partial_{\nu} u_{\mu}\right)+\left(u_{\mu} \partial_{\nu} \mu_{5}-u_{\nu} \partial_{\mu} \mu_{5}\right),
$$

where we identify two terms respectively related to the vorticity tensor and the spacetime variations of the chemical potential $\mu_{5}$ governing chiral imbalance. For the vector gauge fields $\mathcal{V}_{a \mu}$, we find $(a=0,3)$

$$
\mathcal{V}_{a \mu}=-e^{-\sigma} V_{a 0} u_{\mu}+\delta_{\mu}^{i} V_{a i}=-\mu_{a} u_{\mu}+\delta_{\mu}^{i} V_{a i},
$$

whose field strength tensor, besides the vorticity and chemical potential gradient contributions pointed out in the axial-vector field, has an extra piece

$$
\mathcal{V}_{a \mu \nu}=-\mu_{a}\left(\partial_{\mu} u_{\nu}-\partial_{\nu} u_{\mu}\right)+\left(u_{\mu} \partial_{\nu} \mu_{a}-u_{\nu} \partial_{\mu} \mu_{a}\right)+\delta_{\mu}^{i} \delta_{\nu}^{j} V_{a i j}
$$

with $V_{a i j}=\partial_{i} V_{a j}-\partial_{j} V_{a i}$ the field strength of the KK-invariant components of the Abelian background vector gauge field.

\subsection{Partition function and gauge currents}

At leading (zeroth) order in the derivative expansion, the partition function can be written in terms of the pressure $P_{0}$, which depends on the temperature and the chemical potentials $\mu_{0}$ and $\mu_{3}$ defined in eq. (3.4), namely

$$
W=\frac{1}{T_{0}} \int d^{3} x \sqrt{g} e^{\sigma} P_{0}\left(\mu_{0}, \mu_{3}, T\right)
$$

The first order correction to this result is given by the anomalous equilibrium partition function computed in [32] in the language of differential forms. For a general background, the same expression written in components gives

$$
\begin{aligned}
W\left[V_{0}, A_{0}, V_{i}, A_{i}\right]_{\text {anom }}^{\mathrm{U}(2) \times \mathrm{U}(2)} & \\
= & \frac{N_{c}}{8 \pi^{2} T_{0}} \int_{S^{3}} d^{3} x \sqrt{g} \epsilon^{i j k}\left\{\left(\operatorname{Tr} V_{0}\right) \operatorname{Tr}\left(V_{i j} A_{k}+\frac{4 i}{3} A_{i} A_{j} A_{k}\right)\right. \\
& +\left(\operatorname{Tr} V_{i j}\right) \operatorname{Tr}\left(V_{0} A_{k}\right)+\left[\operatorname{Tr}\left(V_{0} V_{i j}\right)-\left(\operatorname{Tr} V_{0}\right)\left(\operatorname{Tr} V_{i j}\right)\right]\left(\operatorname{Tr} A_{k}\right) \\
& +\frac{1}{3}\left(\operatorname{Tr} A_{i j}\right) \operatorname{Tr}\left(A_{0} A_{k}\right)+\frac{1}{3}\left[\operatorname{Tr}\left(A_{0} A_{i j}\right)-\left(\operatorname{Tr} A_{0}\right)\left(\operatorname{Tr} V_{i j}\right)\right]\left(\operatorname{Tr} A_{k}\right) \\
& +\frac{1}{3}\left(\operatorname{Tr} A_{0}\right) \operatorname{Tr}\left(A_{i j} A_{k}\right)-\frac{4}{3}\left(\operatorname{Tr} A_{i}\right) \operatorname{Tr}\left(V_{0} A_{j} A_{k}\right)
\end{aligned}
$$




$$
\begin{aligned}
& -\frac{1}{2} f_{i j}\left[\left(\operatorname{Tr} V_{0}\right)^{2}-\operatorname{Tr} V_{0}^{2}\right]\left(\operatorname{Tr} A_{k}\right)-\frac{1}{6} f_{i j}\left[\left(\operatorname{Tr} A_{0}\right)^{2}-\operatorname{Tr} A_{0}^{2}\right]\left(\operatorname{Tr} A_{k}\right) \\
& \left.+f_{i j}\left(\operatorname{Tr} V_{0}\right) \operatorname{Tr}\left(V_{0} A_{k}\right)+\frac{1}{3} f_{i j}\left(\operatorname{Tr} A_{0}\right) \operatorname{Tr}\left(A_{0} A_{k}\right)\right\} \\
& +\frac{1}{2} C_{2} T_{0} \int_{S^{3}} d^{3} \mathbf{x} \sqrt{g} \epsilon^{i j k} f_{i j}\left(\operatorname{Tr} A_{k}\right) .
\end{aligned}
$$

Here $\epsilon^{i j k}$ represents the Levi-Civita pseudotensor, normalized according to $\epsilon^{123}=1 / \sqrt{g}$. The last term in the anomalous effective action mixes the axial-vector gauge field with the KK vector field and is responsible for the corrections to the chiral vortical effect quadratic in the temperature. Various analyses show $[25,44,45]$ that the coefficient $C_{2}$ is related to the value of the mixed gauge-gravitational anomaly and is not computable using the differential geometry methods used here. We stress that the functional given in eq. (3.10) only contains KK-invariant gauge fields, while its explicit dependence on the metric function $a_{i}$ comes through the Abelian field strength

$$
f_{i j} \equiv \partial_{i} a_{j}-\partial_{j} a_{i}
$$

Thus, the equilibrium partition function is explicitly invariant under KK gauge transformations.

The covariant vector and axial-vector currents can be computed using eqs. (2.32) and (2.34), particularized to the background (3.2). Expressing them in terms of the fourvelocity $u^{\mu}$, the chemical potentials (3.3) and (3.4), and the field strength components $\mathcal{V}_{\mu \nu}=\mathcal{V}_{a \mu \nu} t_{a}$ of the KK-variant fields [see (2.28)], we arrive at

$$
\begin{aligned}
\left\langle\mathcal{J}_{0 V}^{\mu}\right\rangle_{\text {cov }} & =\frac{N_{c}}{16 \pi^{2}} \mu_{5} \epsilon^{\mu \nu \alpha \beta} u_{\nu} \mathcal{V}_{0 \alpha \beta}, \\
\left\langle\mathcal{J}_{3 V}^{\mu}\right\rangle_{\text {cov }} & =\frac{N_{c}}{16 \pi^{2}} \mu_{5} \epsilon^{\mu \nu \alpha \beta} u_{\nu} \mathcal{V}_{3 \alpha \beta}, \\
\left\langle\mathcal{J}_{0 A}^{\mu}\right\rangle_{\text {cov }} & =\frac{N_{c}}{16 \pi^{2}} \epsilon^{\mu \nu \alpha \beta} u_{\nu}\left[\mu_{0} \mathcal{V}_{0 \alpha \beta}+\mu_{3} \mathcal{V}_{3 \alpha \beta}+\left(\mu_{0}^{2}+\mu_{3}^{2}-\mu_{5}^{2}\right) \partial_{\alpha} u_{\beta}\right], \\
\left\langle\mathcal{J}_{3 A}^{\mu}\right\rangle_{\text {cov }} & =\frac{N_{c}}{16 \pi^{2}} \epsilon^{\mu \nu \alpha \beta} u_{\nu}\left(\mu_{3} \mathcal{V}_{0 \alpha \beta}+\mu_{0} \mathcal{V}_{3 \alpha \beta}+2 \mu_{0} \mu_{3} \partial_{\alpha} u_{\beta}\right)
\end{aligned}
$$

where we have followed the notation introduced in eq. (2.30). In these expressions and in the following, the four-dimensional Levi-Civita pseudotensor is normalized such that ${ }^{3}$

$$
\epsilon^{0123}=\frac{1}{\sqrt{-G}}=\frac{e^{-\sigma}}{\sqrt{g}} \quad \Longrightarrow \quad \epsilon^{0 i j k}=e^{-\sigma} \epsilon^{i j k}
$$

with $G$ the determinant of the static metric (2.4) and $\epsilon^{i j k}$ the three-dimensional antisymmetric pseudotensor normalized as $\epsilon^{123}=1 / \sqrt{g}$ [cf. eq. (3.10)].

The second quantity to be conside is the anomalous contribution to the energymomentum tensor, codified in the vector $q^{\mu}$ defined by

$$
T^{\mu \nu}=u^{\mu} q^{\nu}+u^{\nu} q^{\mu}
$$

\footnotetext{
${ }^{3}$ In this work, we depart from the conventions of ref. [36], where the Levi-Civita tensor is defined with an additional power of $\sqrt{-G}$.
} 
This can be evaluated from eq. (2.38) and takes the form

$$
q^{\mu}=\frac{N_{c}}{16 \pi^{2}} \epsilon^{\mu \nu \alpha \beta} u_{\nu} \mu_{5}\left[\mu_{0} \mathcal{V}_{0 \alpha \beta}+\mu_{3} \mathcal{V}_{3 \alpha \beta}+\left(\mu_{0}^{2}+\mu_{3}^{2}-\frac{1}{3} \mu_{5}^{2}\right) \partial_{\alpha} u_{\beta}\right]
$$

These Abelianized expressions match the general structure for the anomalous currents derived in ref. [46]. It should be pointed out that the vectors in eqs. (3.12) and (3.15) are transverse to $u^{\mu}$, as it follows from the general structure of the covariant currents found in eq. (2.34). As we will see in section 5.3, this property is broken in the presence of NG bosons, where the covariant currents develop a longitudinal component.

Inspecting the different terms in the currents and energy-momentum tensor given in eqs. (3.12) and (3.15), we find that the coupling to the external gauge fields comes through the magnetic components of the non-Abelian vector field strength $(a=0,3)$

$$
\begin{aligned}
\mathcal{B}_{a}^{\mu} \equiv \frac{1}{2} \epsilon^{\mu \nu \alpha \beta} u_{\nu} \mathcal{V}_{a \alpha \beta} & =\left(-a_{j} \epsilon^{j k \ell} \partial_{k} \mathcal{V}_{a \ell}, \epsilon^{i j k}\left(\partial_{j} \mathcal{V}_{a k}+a_{j} \partial_{k} \mathcal{V}_{a 0}\right)\right) \\
& =\left(-a_{j} \epsilon^{j k \ell}\left(\partial_{k} V_{a \ell}+\frac{1}{2} V_{a 0} f_{k \ell}\right), \epsilon^{i j k}\left(\partial_{j} V_{a k}+\frac{1}{2} V_{a 0} f_{j k}\right)\right),
\end{aligned}
$$

where the second line is obtained by applying the decomposition (2.15) to the definition of the magnetic field. The explicit dependence on the four-velocity $u_{\mu}$, on the other hand, is codified in terms of the vorticity vector

$$
\omega^{\mu} \equiv \frac{1}{2} \epsilon^{\mu \nu \alpha \beta} u_{\nu} \partial_{\alpha} u_{\beta}=\frac{1}{4} e^{\sigma}\left(\epsilon^{j k \ell} a_{j} f_{k \ell},-\epsilon^{i j k} f_{j k}\right) .
$$

Having identified these transverse structures, we write the constitutive relations in covariant form

$$
\begin{aligned}
\left\langle\mathcal{J}_{a V}^{\mu}\right\rangle_{\mathrm{cov}} & =\xi_{V, B}^{a b} \mathcal{B}_{b}^{\mu}+\xi_{V}^{a} \omega^{\mu} \\
\left\langle\mathcal{J}_{a A}^{\mu}\right\rangle_{\mathrm{cov}} & =\xi_{A, B}^{a b} \mathcal{B}_{b}^{\mu}+\xi_{A}^{a} \omega^{\mu} \\
q^{\mu} & =\xi_{\epsilon, B}^{a} \mathcal{B}_{a}^{\mu}+\xi_{\epsilon, V} \omega^{\mu}
\end{aligned}
$$

so the corresponding out-of-equilibrium anomalous transport coefficients associated with the chiral magnetic, the chiral vortical, and the chiral separation effects can be read from eqs. (3.12) and (3.15), to give

$$
\begin{array}{rlrl}
\xi_{V, B}^{a b} & =\frac{N_{c}}{8 \pi^{2}} \mu_{5} \delta^{a b}, & \xi_{V}^{a} & =0, \\
\xi_{A, B}^{a b} & =\frac{N_{c}}{8 \pi^{2}}\left[\left(\mu_{0}-\mu_{3}\right) \delta^{a b}+\mu_{3}\right], & \xi_{A}^{a} & =\frac{N_{c}}{8 \pi^{2}}\left[\left(\mu_{0}^{2}+\mu_{3}^{2}-\mu_{5}^{2}\right) \delta^{a 0}+2 \mu_{0} \mu_{3} \delta^{a 3}\right], \\
\xi_{\epsilon, B}^{a}=\frac{N_{c}}{8 \pi^{2}} \mu_{5}\left(\mu_{0} \delta^{a 0}+\mu_{3} \delta^{a 3}\right), & \xi_{\epsilon, V}=\frac{N_{c}}{8 \pi^{2}} \mu_{5}\left(\mu_{0}^{2}+\mu_{3}^{2}-\frac{1}{3} \mu_{5}^{2}\right) .
\end{array}
$$

\subsection{Baryon, isospin, and electromagnetic currents}

We define now the vector baryonic and isospin currents as those associated with the generators $t_{0}$ and $t_{3}$, which properly normalized read

$$
\begin{aligned}
\mathcal{J}_{\text {bar }}^{\mu} & =\frac{2}{3} \bar{\Psi} \gamma^{\mu} t_{0} \Psi, \\
\mathcal{J}_{\text {iso }}^{\mu} & =\bar{\Psi} \gamma^{\mu} t_{3} \Psi,
\end{aligned}
$$


where $\Psi$ is a flavor doublet of Dirac spinors made out of the two light quark flavors

$$
\Psi=\left(\begin{array}{l}
u \\
d
\end{array}\right)
$$

with each spinor being decomposed into its two chiralities according to

$$
u=\left(\begin{array}{c}
u_{L} \\
u_{R}
\end{array}\right), \quad d=\left(\begin{array}{c}
d_{L} \\
d_{R}
\end{array}\right) .
$$

Electromagnetism, on the other hand, is identified with the $\mathrm{U}(1)_{V}$ subgroup generated by the charge matrix $Q$ defined by the Gell-Mann-Nishijima formula

$$
Q \equiv \frac{1}{3} t_{0}+t_{3}=\left(\begin{array}{cc}
\frac{2}{3} & 0 \\
0 & -\frac{1}{3}
\end{array}\right)
$$

where charges are expressed in units of the elementary charge $e$. In terms of $Q$, the electromagnetic current is now given by

$$
\mathcal{J}_{\mathrm{em}}^{\mu} \equiv e \bar{\Psi} \gamma^{\mu} Q \Psi=\frac{e}{3} \bar{\Psi} \gamma^{\mu} t_{0} \Psi+e \bar{\Psi} \gamma^{\mu} t_{3} \Psi
$$

where we have introduced the explicit dependence on $e$.

Since the electromagnetic field is the only propagating gauge field in the theory, it is convenient to take $Q$ as one of the elements of the basis of the Cartan subalgebra of the vector factors $\mathrm{SU}(2)_{V} \times \mathrm{U}(1)_{V}$, instead of the $\mathrm{U}(1)_{B}$ generator $t_{0}$. Then, the vector background field $V_{\mu}$ can be written as

$$
V_{\mu}=V_{0 \mu} t_{0}+V_{3 \mu} t_{3}=3 V_{0 \mu} Q+\left(V_{3 \mu}-3 V_{0 \mu}\right) t_{3} .
$$

We thus identify the electromagnetic potential as the gauge field coupling to $Q$, while the component along the generator of the Cartan subalgebra of $\mathrm{SU}(2)_{V}$ is set to zero, since it is just an auxiliary field which does not contain propagating degrees of freedom at low energies

$$
\left.\begin{array}{rl}
3 V_{0 \mu} & \equiv e \mathbb{V}_{\mu} \\
V_{3 \mu}-3 V_{0 \mu} & =0
\end{array}\right\} \quad \Longrightarrow \quad V_{3 \mu}=3 V_{0 \mu}=e \mathbb{V}_{\mu}
$$

This in turn implies the following relation between the isospin and baryonic chemical potentials

$$
\mu_{0}=\frac{1}{3} \mu_{3}=\frac{e}{3} e^{-\sigma} \bigvee_{0}
$$

At the quantum level, the expectation values of the consistent electromagnetic, baryonic, and isospin currents can be written in terms of the values of the consistent vector currents $\left\langle\mathcal{J}_{a V}^{\mu}\right\rangle_{\text {cons }}$ (with $a=0,3$ ), obtained by varying the quantum effective action

$$
\begin{aligned}
\left\langle\mathcal{J}_{\text {em }}^{\mu}\right\rangle_{\text {cons }} & =\frac{e}{3}\left\langle\mathcal{J}_{0 V}^{\mu}\right\rangle_{\text {cons }}+e\left\langle\mathcal{J}_{3 V}^{\mu}\right\rangle_{\text {cons }}, \\
\left\langle\mathcal{J}_{\text {bar }}^{\mu}\right\rangle_{\text {cons }} & =\frac{2}{3}\left\langle\mathcal{J}_{0 V}^{\mu}\right\rangle_{\text {cons }}, \\
\left\langle\mathcal{J}_{\text {iso }}^{\mu}\right\rangle_{\text {cons }} & =\left\langle\mathcal{J}_{3 V}^{\mu}\right\rangle_{\text {cons }} .
\end{aligned}
$$


The same pattern is followed by the BZ terms of each current and, as a consequence, the same relations are satisfied by the corresponding covariant currents. Given these expressions, it is clear that the currents defined in eqs. (3.20) and (3.24) satisfy the Gell-MannNishijima relation also at the quantum level

$$
\left\langle\mathcal{J}_{\mathrm{em}}^{\mu}\right\rangle=\frac{e}{2}\left\langle\mathcal{J}_{\text {bar }}^{\mu}\right\rangle+e\left\langle\mathcal{J}_{\text {iso }}^{\mu}\right\rangle .
$$

We can use now the constitutive relations (3.18) and transport coefficients (3.19) to find the constitutive relations for the covariant electromagnetic current

$$
\begin{aligned}
\left\langle\mathcal{J}_{\mathrm{em}}^{\mu}\right\rangle_{\mathrm{cov}} & =\frac{e}{3}\left\langle\mathcal{J}_{0 V}^{\mu}\right\rangle_{\mathrm{cov}}+e\left\langle\mathcal{J}_{3 V}^{\mu}\right\rangle_{\mathrm{cov}} \\
& =\frac{e N_{c}}{24 \pi^{2}} \mu_{5}\left(\mathcal{B}_{0}^{\mu}+3 \mathcal{B}_{3}^{\mu}\right) .
\end{aligned}
$$

To recast the term inside the bracket in terms of the physical electromagnetic field, we recall eq. (3.26), from where the following expression of the KK-variant components of the electromagnetic potential $\mathcal{V}_{\mu}$ follows

$$
\left(\mathcal{V}_{0}, \mathcal{V}_{i}\right) \equiv\left(\mathbb{V}_{0}, \mathbb{V}_{i}+\mathbb{V}_{0} a_{i}\right) \quad \Longrightarrow \quad\left\{\begin{array}{l}
\mathcal{E}_{3}^{\mu}=3 \mathcal{E}_{0}^{\mu}=e \mathcal{E}^{\mu} \\
\mathcal{B}_{3}^{\mu}=3 \mathcal{B}_{0}^{\mu}=e \mathcal{B}^{\mu}
\end{array}\right.
$$

where the KK-variant electric and magnetic fields are defined in the usual way from the corresponding field strength

$$
\begin{aligned}
\mathcal{E}_{\mu} & \equiv \mathcal{V}_{\mu \nu} u^{\nu}, \\
\mathcal{B}^{\mu} & \equiv \frac{1}{2} \epsilon^{\mu \nu \alpha \beta} u_{\nu} \mathcal{V}_{\alpha \beta} .
\end{aligned}
$$

With this, the constitutive equation for the electromagnetic current in eq. (3.30) reads

$$
\left\langle\mathcal{J}_{\mathrm{em}}^{\mu}\right\rangle_{\mathrm{cov}}=\frac{5 e^{2} N_{c}}{36 \pi^{2}} \mu_{5} \mathcal{B}^{\mu},
$$

which also gives the transport coefficient associated with the chiral magnetic effect. On the other hand, the vanishing of the coefficient $\xi_{V}^{a}$ implies the absence of a chiral vortical effect in the vector currents of the unbroken theory.

Alternatively, we can express the current in terms of the KK-invariant magnetic field defined from the electromagnetic potential $\mathbb{V}_{\mu}$

$$
\mathbb{B}^{\mu} \equiv \mathcal{B}^{\mu}+\frac{6}{e} \mu_{0} \omega^{\mu}=\left(-a_{j} \epsilon^{j k \ell} \partial_{k} \vee_{\ell}, \epsilon^{i j k} \partial_{j} \vee_{k}\right)
$$

to read

$$
\left\langle\mathcal{J}_{\mathrm{em}}^{\mu}\right\rangle_{\mathrm{cov}}=\frac{5 e^{2} N_{c}}{36 \pi^{2}} \mu_{5} \mathbb{B}^{\mu}-\frac{5 e N_{c}}{6 \pi^{2}} \mu_{0} \mu_{5} \omega^{\mu}
$$

Thus, once expressed in terms of the physical fields, a contribution to the chiral vortical conductivity emerges, together with the one associated with the chiral magnetic effect. This is similar to the results found in [47] for an Abelian $\mathrm{U}(1)_{V} \times \mathrm{U}(1)_{A}$ theory. 
The cancellation leading to our result $\xi_{V}^{a}=0$ in (3.19) is a direct consequence of having considered the flavor group $\mathrm{U}(2) \times \mathrm{U}(2)$ and, to our knowledge, has not been noticed before in the literature. In a sense, this means that when expressed in terms of the KKinvariant fields the chiral vortical effect in eq. (3.35) is geometrically determined, since its value is encoded in the relation between KK-variant and KK-invariant gauge fields components (2.13). This state of affairs contrasts with the $\mathrm{U}(1) \times \mathrm{U}(1)$ case, studied in $[47$, 48], where such cancellation does not take place.

\section{Pion partition function of two-flavor QCD in the chiral limit}

Let us allow now for the possibility of spontaneous chiral symmetry breaking in the theory studied in the previous section. The first thing to be taken into account is that the axial symmetry $\mathrm{U}(1)_{A}$ is already broken by nonperturbative effects and therefore not realized as an invariance of the theory. As a consequence, we focus on the spontaneous breaking of the surviving subgroups $\mathrm{SU}(2)_{L} \times \mathrm{SU}(2)_{R} \times \mathrm{U}(1)_{V}$ down to its vector-like factors $\mathrm{SU}(2)_{V} \times \mathrm{U}(1)_{V}$. In the process, three NG bosons (the three pions) appear, corresponding to the broken generators of $\mathrm{SU}(2)_{A}$. No NG boson is associated with the $\mathrm{U}(1)_{A}$ factor since, as mentioned, this symmetry is absent from the start.

These NG bosons are codified in the matrix $U$, which can be parametrized using the Pauli matrices $\sigma_{a}$ as

$$
U=\exp \left(i \sum_{a=1}^{3} \zeta_{a} \sigma_{a}\right) \quad \text { with } \quad \sum_{a=1}^{3} \zeta_{a} \sigma_{a}=\frac{\sqrt{2}}{f_{\pi}}\left(\begin{array}{cc}
\frac{1}{\sqrt{2}} \pi^{0} & \pi^{+} \\
\pi^{-} & -\frac{1}{\sqrt{2}} \pi^{0}
\end{array}\right),
$$

with $\pi^{0}, \pi^{ \pm}$the three pion fields and $f_{\pi} \approx 92 \mathrm{MeV}$ the pion decay constant. In order to secure the invariance of the effective action under the gauge transformations of the electromagnetic potential introduced in eq. (3.26), we need the NG field to transform according to

$$
\delta_{\chi} \mathbb{V}_{i}(\mathbf{x})=\partial_{i} \chi(\mathbf{x}) \quad \Longrightarrow \quad \delta_{\chi} U(\mathbf{x})=-\chi(\mathbf{x})[Q, U(\mathbf{x})],
$$

which gives the correct assignment of charges to the three pions.

Having zero baryon number, pions do not couple to the gauge field associated with the baryon number factor $\mathrm{U}(1)_{B}$ generated by $t_{0}$, although they do couple to the gauge field along the isospin generator $t_{3}$. The covariant derivative acting on the NG boson field reads

$$
\xi_{\mu} \equiv D_{\mu} U=\partial_{\mu} U-i \mathcal{V}_{3 \mu}\left[t_{3}, U\right]
$$

where we see how $\mathcal{V}_{0 \mu} t_{0}$ drops out of the commutator. With this in mind, the action at lowest order in derivatives can be written as

$$
\begin{aligned}
W & =\frac{1}{T_{0}} \int d^{3} x \sqrt{g} e^{\sigma} P\left(\mu_{0}, \mu_{3}, T, \mathcal{V}_{3 \mu}, U\right) \\
& =\frac{1}{T_{0}} \int d^{3} x \sqrt{g} e^{\sigma}\left[P_{0}\left(\mu_{0}, \mu_{3}, T\right)+\mathscr{L}\right],
\end{aligned}
$$


where $P_{0}$ is the pressure in the absence of NG bosons, already introduced in eq. (3.9). The second term $\mathscr{L}$ contains all dependence on the pions and has the following expression in terms of KK-invariant fields

$$
\begin{aligned}
\mathscr{L} & =\frac{f_{\pi}^{2}}{4} G^{\mu \nu} \operatorname{Tr}\left[D_{\mu} U\left(D_{\nu} U\right)^{\dagger}\right] \\
& =\frac{f_{\pi}^{2}}{4}\left[e^{-2 \sigma} \operatorname{Tr}\left(\xi_{0} \xi_{0}^{\dagger}\right)-g^{i j} \operatorname{Tr}\left(\Phi_{i} \Phi_{j}^{\dagger}\right)\right],
\end{aligned}
$$

where $\Phi_{i}$ are defined by

$$
\Phi_{i}=\partial_{i} U-i V_{3 i}\left[t_{3}, U\right] .
$$

Notice that in this expression $Q$ can replace $t_{3}$ inside the commutator, whereas from eq. (3.26) we know that $V_{3 i}=e \mathbb{V}_{i}$. Similarly, since $V_{30}=e \mathbb{V}_{0}$, we find that $\xi_{0}=$ $-i e \mathbb{V}_{0}[Q, U]$. As a consequence, the leading order Lagrangian (4.5) governs the electromagnetic coupling of the two charged pions $\pi^{ \pm}$. In the case of the neutral pion, the analogous coupling is mediated by the anomaly and only appears at the next order in the derivative expansion, as we will see below.

At first order in derivatives, the correction to the partition function is given by the corresponding WZW action in the background (3.2). This quantity was computed in [32] and in components reads

$$
\begin{aligned}
W\left[V_{0 \mu},\right. & \left.V_{3 \mu}, A_{00}, U\right]_{\mathrm{WZW}}^{\mathrm{U}(2) \times \mathrm{U}(2)} \\
= & \frac{1}{8 \pi^{2} T_{0}} \int_{S^{3}} d^{3} x \sqrt{g} \epsilon^{i j k}\left\{-\frac{1}{2} V_{00} V_{3 i} \partial_{j} \operatorname{Tr}\left[\left(R_{k}+L_{k}\right) Q\right]\right. \\
& +\frac{i}{6} V_{00} \operatorname{Tr}\left(L_{i} L_{j} L_{k}\right)+\frac{1}{2}\left(V_{00} \partial_{i} V_{3 j}+V_{30} \partial_{i} V_{0 j}+\frac{1}{2} f_{i j} V_{00} V_{30}\right) \operatorname{Tr}\left[\left(R_{k}+L_{k}\right) Q\right] \\
& +\frac{1}{6} A_{00}\left(\partial_{i} V_{3 j}+\frac{1}{2} f_{i j} V_{30}\right) \operatorname{Tr}\left[\left(R_{k}-L_{k}\right) Q\right] \\
& \left.-\frac{1}{3} A_{00}\left(\partial_{i} V_{3 j}+\frac{1}{2} f_{i j} V_{30}\right) V_{3 k} \operatorname{Tr}\left[Q\left(Q-U^{-1} Q U\right)\right]\right\}
\end{aligned}
$$

Here we have introduced the notation

$$
\begin{aligned}
R_{i} & \equiv i U^{-1} \partial_{i} U, \\
L_{i} & \equiv i \partial_{i} U U^{-1} .
\end{aligned}
$$

Notice that since $U$ takes values on $\mathrm{SU}(2)$, the generator $t_{3}$ can be interchanged with the charge matrix $Q$ inside the traces in eq. (4.7).

We can now expand the equilibrium partition function (4.7) in powers of the pion fields. Keeping terms with just one derivative and up to two pion fields, the basics identities to be used are

$$
\begin{aligned}
\operatorname{Tr}\left[\left(R_{i}+L_{i}\right) Q\right] & =-\frac{2}{f_{\pi}} \partial_{i} \pi^{0}+\mathcal{O}\left(\pi^{3}\right), \\
\operatorname{Tr}\left[\left(R_{i}-L_{i}\right) Q\right]+2 \mathbb{V}_{i} \operatorname{Tr}\left[\left(U^{-1} Q U-Q\right) Q\right]= & \frac{2 i}{f_{\pi}^{2}}\left(\partial_{i} \pi^{-} \pi^{+}-\pi^{-} \partial_{i} \pi^{+}\right) \\
& -\frac{4}{f_{\pi}^{2}} \pi^{-} \pi^{+} \bigvee_{i}+\mathcal{O}\left(\pi^{4}\right) .
\end{aligned}
$$


Plugging these expansions into eq. (4.7), we find the following partition function in terms of the pion and electromagnetic field

$$
\begin{aligned}
W\left[\mathbb{V}_{\mu}, \pi^{0}, \pi^{ \pm}\right]_{\mathrm{WZW}}= & -\int_{S^{3}} d^{3} x \sqrt{g}\left[\frac{e^{2} N_{c}}{12 \pi^{2} f_{\pi} T_{0}} \bigvee_{0} \partial_{k} \pi^{0}\left(\mathbb{B}^{k}+\frac{1}{4} \epsilon^{i j k} f_{i j} \bigvee_{0}\right)\right. \\
& \left.+\frac{i \mu_{5} e N_{c}}{24 \pi^{2} f_{\pi}^{2} T}\left(\pi^{-} \partial_{k} \pi^{+}-\pi^{+} \partial_{k} \pi^{-}-2 i e \pi^{+} \pi^{-} \bigvee_{k}\right)\left(\mathbb{B}^{k}+\frac{1}{2} \epsilon^{i j k} f_{i j} \bigvee_{0}\right)\right],
\end{aligned}
$$

where the magnetic field is defined as usual by $\mathbb{B}^{i}=\epsilon^{i j k} \partial_{j} \vee_{k}$ [cf. eq. (3.34)]. In addition, we have used eq. (3.3) to identify the chemical potential governing chiral imbalance, as well as the local temperature $T(\mathbf{x})=T_{0} e^{-\sigma(\mathbf{x})}$. While the first term gives the anomaly-mediated coupling of the neutral pion to the electromagnetic field, corrected by the effect of the curved background, the second one agrees with the form of the parity-odd couplings obtained from the WZW effective action in systems with chiral imbalance, as shown in [49, 50].

To compare with existing results in the literature, it is convenient to go back to (4.7) and recast it in terms of the electromagnetic potential $\mathbb{V}_{\mu}$ as

$$
\begin{aligned}
W\left[\mathbb{V}_{\mu}, U\right]_{\mathrm{WZW}}^{\mathrm{U}(2) \times \mathrm{U}(2)} \\
=\int_{S^{3}} d^{3} x \sqrt{g} \epsilon^{i j k}\left(g_{1}(\nu) \partial_{i} \vee_{j} \operatorname{Tr}\left[Q\left(R_{k}+L_{k}\right)\right]\right. \\
\quad+g_{2}(\nu)\left\{-\frac{1}{2} \mathbb{\vee}_{i} \operatorname{Tr}\left[Q \partial_{j}\left(R_{k}+L_{k}\right)\right]+\frac{i}{6 e} \operatorname{Tr}\left(L_{i} L_{j} L_{k}\right)\right\}+\frac{T_{0}}{2} g_{3}(\nu) f_{i j} \operatorname{Tr}\left[Q\left(R_{k}+L_{k}\right)\right] \\
\left.\quad+g_{4}\left(\nu_{5}\right)\left(\partial_{i} \vee_{j}+\frac{1}{2} f_{i j} \vee_{0}\right)\left\{2 \mathbb{\vee}_{k} \operatorname{Tr}\left[Q\left(Q-U^{-1} Q U\right)\right]-\frac{1}{e} \operatorname{Tr}\left[Q\left(R_{k}-L_{k}\right)\right]\right\}\right),
\end{aligned}
$$

where we have written

$$
\begin{gathered}
\nu(\mathbf{x}) \equiv \frac{\mu(\mathbf{x})}{T(\mathbf{x})}=\frac{\mathbb{V}_{0}(\mathbf{x})}{T_{0}}, \\
\nu_{5}(\mathbf{x}) \equiv \frac{\mu_{5}(\mathbf{x})}{T(\mathbf{x})}=\frac{A_{00}(\mathbf{x})}{T_{0}},
\end{gathered}
$$

the chemical potential $\mu=e^{-\sigma} \bigvee_{0}$ being associated with electric charge imbalance. Our microscopic computation of the non-Abelian anomalous effective action leads to the following prediction for the couplings

$$
\begin{aligned}
g_{1}(\nu) & =g_{2}(\nu)=\frac{N_{c} e^{2}}{24 \pi^{2}} \nu(\mathbf{x}) \\
g_{3}(\nu) & =\frac{N_{c} e^{2}}{48 \pi^{2}} \nu(\mathbf{x})^{2} \\
g_{4}\left(\nu_{5}\right) & =\frac{N_{c} e^{2}}{48 \pi^{2}} \nu_{5}(\mathbf{x}),
\end{aligned}
$$

where the corresponding operators are gauge invariant. The equilibrium partition function (4.11) is the analog to eq. (2.26) of ref. [51], where the couplings (4.13) parametrize the action of a single NG boson associated with a broken U(1) symmetry. Similar formulae have been obtained in ref. [52]. Notice, however, that our expression is more general. In particular, it allows the computation of currents in sectors different from the electromagnetic one, as it will be shown later. 


\section{Constitutive relations of the two-flavor hadronic superfluid}

The covariant currents computed above provide the first correction in the derivative expansion of the hydrodynamics constitutive relations for the currents and the energy-momentum tensor. On general grounds, physical quantities in hydrodynamics admit the decomposition in terms of their perfect fluid contributions and corrections containing higher terms in derivatives. For the case of a generic current and the energy-momentum tensor, we have [51-54]

$$
\begin{aligned}
\mathcal{J}^{\mu} & =\mathcal{J}_{\mathrm{PF}}^{\mu}+\nu^{\mu}, \\
T^{\mu \nu} & =T_{\mathrm{PF}}^{\mu \nu}+\Pi^{\mu \nu},
\end{aligned}
$$

where the subscripts PF indicate the perfect fluid contributions and $\nu^{\mu}$ and $\Pi^{\mu \nu}$ are written in terms of the tensor quantities characterizing the system, as well as their spacetime derivatives. The constitutive relations provide the dependence of the coefficients of each term on physical quantities such as the temperature and the chemical potentials. Nonperfect fluids terms in (5.1) induce corrections in these constitutive relations at first and higher-order in derivatives.

The presence of these gradient-dependent corrections to the perfect fluid quantities leads to ambiguities, since different definitions of the same physical variable may vary by gradient-dependent terms $\left(\delta T, \delta \mu, \delta u^{\mu}, \ldots\right)$ which vanish in the limit of ideal hydrodynamics. Since these ambiguities cannot change the form of the currents and the energymomentum tensor, the perfect fluid constitutive relations have to compensate these higher order terms by a shift in their perfect fluid values

$$
\begin{aligned}
& T_{0} \longrightarrow T_{0}+\delta T(x), \\
& \mu_{0} \longrightarrow \mu_{0}+\delta \mu(x), \\
& u_{0}^{\mu} \longrightarrow u_{0}^{\mu}+\delta u^{\mu}(x) .
\end{aligned}
$$

In hydrodynamics, these kind of ambiguities are fixed by selecting a frame, which in our case is chosen by requiring that one-derivative corrections to perfect fluid quantities vanish. As a consequence, constributions at this order come only from the terms $\nu^{\mu}$ and $\Pi^{\mu \nu}$ in eq. (5.1). For systems with spontaneous symmetry breaking, it was found [32] that the energy-momentum tensor receives no corrections

$$
\Pi^{\mu \nu}=0 .
$$

There are nevertheless nonvanishing corrections $\nu^{\mu}$ to the current, which admit the decomposition (2.16) in terms of its longitudinal and transverse components

$$
\nu^{\mu}=e^{\sigma}\left(\nu^{0}+a_{i} \nu^{i}\right) u^{\mu}+\left(\delta_{i}^{\mu}-\delta_{0}^{\mu} a_{i}\right) \nu^{i} .
$$

As explained in section 2, the combinations $\left(\nu^{0}+a_{j} \nu^{j}, \nu^{i}\right)$ are KK-invariant. 


\subsection{The gauge currents at leading order}

After these general considerations, we go back to the spontaneously broken two-flavor QCD model presented above, and begin with the analysis of the gauge currents at leading order in the derivative expansion. These and the energy-momentum tensor are computed by taking the corresponding functional derivatives on the effective action (4.4). For the gauge currents, this calculation leads to the expressions ${ }^{4}$

$$
\begin{aligned}
\left\langle\mathcal{J}_{00}\right\rangle_{\mathrm{PF}} & \equiv-\frac{T_{0} e^{\sigma}}{\sqrt{g}} \frac{\delta W}{\delta V_{00}}=-n_{0} e^{\sigma}, \\
\left\langle\mathcal{J}_{30}\right\rangle_{\mathrm{PF}} & \equiv-\frac{T_{0} e^{\sigma}}{\sqrt{g}} \frac{\delta W}{\delta V_{30}}=-n_{3} e^{\sigma}+\frac{f_{\pi}^{2}}{2} V_{30} \operatorname{Tr}\left([Q, U]\left[Q, U^{\dagger}\right]\right), \\
\left\langle\mathcal{J}_{0}^{i}\right\rangle_{\mathrm{PF}} & \equiv \frac{T_{0} e^{-\sigma}}{\sqrt{g}} \frac{\delta W}{\delta V_{0 i}}=0, \\
\left\langle\mathcal{J}_{3}^{i}\right\rangle_{\mathrm{PF}} & \equiv \frac{T_{0} e^{-\sigma}}{\sqrt{g}} \frac{\delta W}{\delta V_{3 i}}=\frac{i f_{\pi}^{2}}{4} g^{i j} \operatorname{Tr}\left([Q, U] \Phi_{j}^{\dagger}+\left[Q, U^{\dagger}\right] \Phi_{j}\right),
\end{aligned}
$$

where the number densities $n_{0}$ and $n_{3}$ are defined by

$$
n_{0}=\frac{\partial P}{\partial \mu_{0}}, \quad n_{3}=\frac{\partial P}{\partial \mu_{3}} .
$$

These densities satisfy the thermodynamic identity

$$
\varepsilon+P=T s+\mu_{0} n_{0}+\mu_{3} n_{3},
$$

where the entropy density is given by

$$
s=\frac{\partial P}{\partial T} .
$$

In computing the currents (5.5) we have also replaced $t_{3}$ by $Q$ inside the commutators.

It is interesting to notice that, since the term $\mathscr{L}$ in (4.4) does not depend on $V_{0 \mu}$, it does not contribute to the baryonic current. Then, the relations (3.28) imply that the NG-dependent part of the consistent electromagnetic current is completely determined by the isospin one. On the other hand, the NG-independent term $P_{0}$ would contribute to both the baryonic and isospin currents, since this function depends on both $V_{00}$ and $V_{30}$. Finally, the components of the energy-momentum tensor are similarly computed to give

$$
\begin{aligned}
\left\langle T_{00}\right\rangle_{\mathrm{PF}} & \equiv-\frac{T_{0} e^{\sigma}}{\sqrt{g}} \frac{\delta W}{\delta \sigma}=e^{2 \sigma} \varepsilon+\frac{f_{\pi}^{2}}{2} \operatorname{Tr}\left(\xi_{0} \xi_{0}^{\dagger}\right), \\
\left\langle T_{0}{ }^{i}\right\rangle_{\mathrm{PF}} & \equiv \frac{T_{0} e^{-\sigma}}{\sqrt{g}}\left(\frac{\delta W}{\delta a_{i}}-V_{00} \frac{\delta W}{\delta V_{0 i}}-V_{30} \frac{\delta W}{\delta V_{3 i}}\right)=\frac{f_{\pi}^{2}}{4} g^{i j} \operatorname{Tr}\left(\xi_{0} \Phi_{j}^{\dagger}+\xi_{0}^{\dagger} \Phi_{j}\right), \\
\left\langle T^{i j}\right\rangle_{\mathrm{PF}} & \equiv-\frac{2 T_{0} e^{\sigma}}{\sqrt{g}} g^{i k} g^{j m} \frac{\delta W}{\delta g^{k m}}=P g^{i j}+\frac{f_{\pi}^{2}}{4} g^{i k} g^{j m} \operatorname{Tr}\left(\Phi_{k} \Phi_{m}^{\dagger}+\Phi_{m} \Phi_{k}^{\dagger}\right) .
\end{aligned}
$$

\footnotetext{
${ }^{4}$ Since the BZ terms contain one derivative of the gauge fields (see section 5.2), at leading order in the derivative expansion there is no distinction between consistent and covariant currents. The subscript in the expectation value just indicates that they provide the perfect fluid contribution.
} 
These expressions can be brought into a covariant form by writing them in terms of the static metric $G_{\mu \nu}$ defined in eq. (2.4) and the four velocity $u_{\mu}$ given in (2.7). For the currents, we find

$$
\begin{aligned}
\left\langle\mathcal{J}_{0 \mu}\right\rangle_{\mathrm{PF}}= & n_{0} u_{\mu}, \\
\left\langle\mathcal{J}_{3 \mu}\right\rangle_{\mathrm{PF}}= & n_{3} u_{\mu}+\frac{i f_{\pi}^{2}}{4} \operatorname{Tr}\left([Q, U] \partial_{\mu} U^{\dagger}+\left[Q, U^{\dagger}\right] \partial_{\mu} U\right) \\
& +\frac{f_{\pi}^{2}}{2} V_{3 \mu} \operatorname{Tr}\left([Q, U]\left[Q, U^{\dagger}\right]\right),
\end{aligned}
$$

whereas the expression for the energy-momentum tensor is

$$
\left\langle T^{\mu \nu}\right\rangle_{\mathrm{PF}}=(\epsilon+P) u^{\mu} u^{\nu}+P G^{\mu \nu}+\frac{f_{\pi}^{2}}{4} G^{\mu \alpha} G^{\nu \beta} \operatorname{Tr}\left[D_{\alpha} U\left(D_{\beta} U\right)^{\dagger}+D_{\beta} U\left(D_{\alpha} U\right)^{\dagger}\right] .
$$

\subsection{Covariant currents and Bardeen-Zumino terms at first order in derivatives}

The anomaly-induced WZW partition function (4.7) provides the corrections at first order in derivatives to the leading order currents and energy-momentum tensor given in eqs. (5.5) and (5.9). It is at this order that we have to start distinguishing between consistent and covariant currents. The usual approach to compute them is to begin with the consistent current, which is obtained by taking functional derivatives of the WZW partition function (4.7). The covariant current is then found by adding the corresponding BZ terms. Here we use a more efficient procedure developed in [32] and reviewed in section 2. Instead of using the WZW action, we arrive at the covariant current directly from the BZ current using eqs. (2.50) and (2.52).

The fact that the anomaly admits two different forms, consistent and covariant, brings up the issue of which current is physically relevant for the evaluation of the anomalous transport coefficients. As far as the physics of transport is concerned, it is necessary to consider the covariant currents, as can be argued using the notion of anomaly inflow [55]. As seen in $[24,27,32]$, the covariant anomaly is the result of the transport of conserved global charge in the higher-dimensional bulk space that overflows onto the spacetime boundary. This strongly indicates that actual charge transport is governed by the covariant current, while the consistent current determine the coupling to the sources in the equilibrium partition function. ${ }^{5}$

The upshot is that covariant currents, although not coupling directly to the external fields, are the ones relevant in the hydrodynamic description and provide the constitutive relations that lead to the determination of the different transport coefficients. In the case at hand, we particularize eq. (2.50) to the background defined in (3.2), keeping always in mind that $A_{00}=e^{\sigma} \mu_{5}$ is a constant field. Then, we apply the relations (2.52) leading to the calculation of the vector and axial-vector components of the current. After some algebra, we arrive at

$$
\begin{aligned}
\left\langle\mathcal{J}_{0 V}^{\mu}\right\rangle_{\text {cov }}=-\frac{N_{c}}{16 \pi^{2}} \epsilon^{\mu \nu \alpha \beta}\{ & \mathcal{A}_{0 \nu} \mathcal{V}_{0 \alpha \beta}-\frac{1}{2} \mathcal{V}_{3 \nu \alpha} \operatorname{Tr}\left[\left(R_{\beta}+L_{\beta}\right) Q\right] \\
& \left.+\mathcal{V}_{3 \nu} \partial_{\alpha} \operatorname{Tr}\left[\left(R_{\beta}+L_{\beta}\right) Q\right]-\frac{i}{3} \operatorname{Tr}\left(L_{\nu} L_{\alpha} L_{\beta}\right)\right\},
\end{aligned}
$$

\footnotetext{
${ }^{5}$ The physical relevance of the covariant current in a different context has been discussed in [56].
} 


$$
\begin{aligned}
\left\langle\mathcal{J}_{3 V}^{\mu}\right\rangle_{\text {cov }}=-\frac{N_{c}}{48 \pi^{2}} \epsilon^{\mu \nu \alpha \beta} & \left(3 \mathcal{A}_{0 \nu} \mathcal{V}_{3 \alpha \beta}+\mathcal{A}_{0 \nu} \mathcal{V}_{3 \alpha \beta} \operatorname{Tr}\left[\left(U^{-1} Q U-Q\right) Q\right]\right. \\
& -\partial_{\alpha}\left\{\mathcal{A}_{0 \nu} \operatorname{Tr}\left[\left(R_{\beta}-L_{\beta}\right) Q\right]+2 \mathcal{A}_{0 \nu} \mathcal{V}_{3 \beta} \operatorname{Tr}\left[\left(U^{-1} Q U-Q\right) Q\right]\right\} \\
& \left.-\frac{3}{2} \mathcal{V}_{0 \nu \alpha} \operatorname{Tr}\left[\left(R_{\beta}+L_{\beta}\right) Q\right]\right),
\end{aligned}
$$

for the vector currents. In the case of the axial-vector currents, a similar calculation leads to the results

$$
\begin{aligned}
\left\langle\mathcal{J}_{0 A}^{\mu}\right\rangle_{\text {cov }}=-\frac{N_{c}}{48 \pi^{2}} \epsilon^{\mu \nu \alpha \beta} & \left(\mathcal{A}_{0 \nu} \mathcal{A}_{0 \alpha \beta}-\frac{1}{2} \mathcal{V}_{3 \nu \alpha}\left\{\operatorname{Tr}\left[\left(R_{\beta}-L_{\beta}\right) Q\right]\right.\right. \\
& \left.\left.+2 \mathcal{V}_{3 \beta} \operatorname{Tr}\left[\left(U^{-1} Q U-Q\right) Q\right]\right\}\right) \\
\left\langle\mathcal{J}_{3 A}^{\mu}\right\rangle_{\text {cov }}=-\frac{N_{c}}{48 \pi^{2}} \epsilon^{\mu \nu \alpha \beta} & \left(-\frac{3}{2} \mathcal{V}_{0 \nu \alpha}\left\{\operatorname{Tr}\left[\left(R_{\beta}-L_{\beta}\right) t_{3}\right]+2 \mathcal{V}_{3 \beta} \operatorname{Tr}\left[\left(U^{-1} Q U-Q\right) Q\right]\right\}\right. \\
& \left.-\frac{1}{2} \mathcal{A}_{0 \nu \alpha} \operatorname{Tr}\left[\left(R_{\beta}+L_{\beta}\right) Q\right]+\mathcal{A}_{0 \nu} \partial_{\alpha} \operatorname{Tr}\left[\left(R_{\beta}+L_{\beta}\right) Q\right]\right) .
\end{aligned}
$$

We will make use of these covariant expressions in later sections to identify the relevant tensor structures and compute the out-of-equilibrium transport coefficients.

In order to find the consistent currents, we need to find the explicit form of the BZ terms (2.46) in the background (3.2). For the vector BZ currents we have $(a=0,3)$

$$
\left\langle\mathcal{J}_{a V}^{\mu}\right\rangle_{\mathrm{BZ}}=-\frac{N_{c}}{4 \pi^{2}} \epsilon^{\mu \nu \alpha \beta} \operatorname{Tr}\left(t_{a} \mathcal{V}_{\nu \alpha} \mathcal{A}_{\beta}\right)=-\frac{N_{c}}{16 \pi^{2}} \epsilon^{\mu \nu \alpha \beta} \mathcal{A}_{0 \nu} \mathcal{V}_{a \alpha \beta}
$$

Using eq. (3.5), it can be written as

$$
\left\langle\mathcal{J}_{a V}^{\mu}\right\rangle_{\mathrm{BZ}}=\frac{N_{c}}{8 \pi^{2}} e^{-\sigma} A_{00} \mathcal{B}_{a}^{\mu}=\frac{N_{c}}{8 \pi^{2}} \mu_{5} \mathcal{B}_{a}^{\mu},
$$

where the magnetic field (3.16) has been identified.

At this point, it is important to stress that since in the background (3.2) all fields lie on the Cartan subalgebra of the group the theory is Abelianized, so the term cubic in $\mathcal{A}_{\mu}$ in the general expression of the BZ current vanishes. We should remember, however, that this formally vanishing cubic term has to be kept when using the BZ current to compute the covariant currents applying (2.47). The reason is that the term gives a nonvanishing contribution upon transforming the left gauge field, as it is required by our prescription. Our construction of the covariant currents presented above used the explicit expression (2.50), which already includes this contribution.

A similar analysis can be carried out for the axial-vector BZ current shown in (2.46), which in the background of interest takes the form

$$
\begin{aligned}
\left\langle\mathcal{J}_{a A}^{\mu}\right\rangle_{\mathrm{BZ}} & =-\frac{N_{c}}{12 \pi^{2}} \epsilon^{\mu \nu \alpha \beta} \operatorname{Tr}\left(t_{a} \mathcal{A}_{\nu} \mathcal{A}_{\alpha \beta}\right)=-\frac{N_{c} \delta_{a 0}}{24 \pi^{2}} \epsilon^{\mu \nu \alpha \beta} \mathcal{A}_{0 \nu} \partial_{\alpha} \mathcal{A}_{0 \beta} \\
& =-\frac{N_{c} \delta_{a 0}}{12 \pi^{2}} e^{-2 \sigma} A_{00}^{2} \omega^{\mu}=-\frac{N_{c} \delta_{a 0}}{12 \pi^{2}} \mu_{5}^{2} \omega^{\mu}
\end{aligned}
$$


where we used the vorticity vector (3.17) and introduced the Kronecker delta to indicate that $\left\langle\mathcal{J}_{3 A}^{\mu}\right\rangle_{\text {BZ vanishes identically }}$

$$
\left\langle\mathcal{J}_{3 A}^{\mu}\right\rangle_{\mathrm{BZ}}=0
$$

Once the BZ currents are known, we compute the consistent gauge currents using

$$
\left\langle\mathcal{J}_{a V, A}^{\mu}\right\rangle_{\mathrm{cons}}=\left\langle\mathcal{J}_{a V, A}^{\mu}\right\rangle_{\mathrm{cov}}-\left\langle\mathcal{J}_{a V, A}^{\mu}\right\rangle_{\mathrm{BZ}}
$$

Subtracting the BZ terms computed in eqs. (5.14) and (5.16) from the covariant currents (5.12) and (5.13), we find that the consistent currents can be identified from the covariant ones just by dropping all terms independent of the NG boson matrix $U$. The consistent currents can be alternatively computed taking functional derivatives from the WZW effective action [37]

$$
\begin{aligned}
\left\langle\mathcal{J}_{a 0 V}\right\rangle_{\text {cons }} & =-\frac{T_{0} e^{\sigma}}{\sqrt{g}} \frac{\delta W}{\delta V_{a 0}}, \\
\left\langle\mathcal{J}_{a V}^{i}\right\rangle_{\text {cons }} & =\frac{T_{0} e^{-\sigma}}{\sqrt{g}} \frac{\delta W}{\delta V_{a i}}, \\
\left\langle\mathcal{J}_{a 0 A}\right\rangle_{\text {cons }} & =-\frac{T_{0} e^{\sigma}}{\sqrt{g}} \frac{\delta W}{\delta A_{a 0}}, \\
\left\langle\mathcal{J}_{a A}^{i}\right\rangle_{\text {cons }} & =\frac{T_{0} e^{-\sigma}}{\sqrt{g}} \frac{\delta W}{\delta A_{a i}} .
\end{aligned}
$$

However, in computing the spatial components of the axial-vector currents, whose dual gauge field are set to zero in the background (3.2), we have to carry out the functional derivatives in the WZW action before actually setting these fields to zero, which notably complicates the calculation. The procedure used here avoids this, since it only makes use of the BZ currents, being much more economical from a computational point of view.

\subsection{Corrections to the leading order constitutive relations}

A look at the results for the vector and axial-vector covariant currents given in eqs. (5.12) and (5.13) shows that all dependence on the NG bosons matrix $U$ comes in terms of the following covariant expressions

$$
\begin{aligned}
\mathbb{H} & \equiv \operatorname{Tr}\left[\left(U^{-1} Q U-Q\right) Q\right] \\
\mathbb{I}_{\mu} & \equiv \operatorname{Tr}\left[\left(R_{\mu}+L_{\mu}\right) Q\right] \\
\mathbb{T}_{\mu} & \equiv \operatorname{Tr}\left[Q\left(R_{\mu}-L_{\mu}\right)\right]+2 \mathcal{V}_{3 \mu} \operatorname{Tr}\left[\left(U^{-1} Q U-Q\right) Q\right] .
\end{aligned}
$$

In terms of them, the one-derivative corrections to the currents in the static background (2.4) take the form

$$
\begin{aligned}
& \left\langle\mathcal{J}_{0 V}^{\mu}\right\rangle_{\text {cov }}=\frac{N_{c}}{16 \pi^{2}} \epsilon^{\mu \nu \alpha \beta}\left[\partial_{\nu} \mathcal{V}_{3 \alpha} \mathbb{I}_{\beta}-\mathcal{V}_{3 \nu} \partial_{\alpha} \mathbb{I}_{\beta}+\frac{i}{3} \operatorname{Tr}\left(L_{\nu} L_{\alpha} L_{\beta}\right)\right]+\frac{N_{c}}{8 \pi^{2}} \mu_{5} \mathcal{B}_{0}^{\mu}, \\
& \left\langle\mathcal{J}_{3 V}^{\mu}\right\rangle_{\text {cov }}=-\frac{N_{c}}{48 \pi^{2}} \epsilon^{\mu \nu \alpha \beta}\left[\partial_{\alpha}\left(\mu_{5} u_{\nu} \mathbb{T}_{\beta}\right)-3 \partial_{\nu} \mathcal{V}_{0 \alpha} \mathbb{I}_{\beta}\right]+\frac{N_{c}}{24 \pi^{2}} \mu_{5}(\mathbb{H}+3) \mathcal{B}_{3}^{\mu},
\end{aligned}
$$


and

$$
\begin{aligned}
& \left\langle\mathcal{J}_{0 A}^{\mu}\right\rangle_{\mathrm{cov}}=\frac{N_{c}}{48 \pi^{2}} \epsilon^{\mu \nu \alpha \beta}\left(\partial_{\nu} \mathcal{V}_{3 \alpha} \mathbb{I}_{\beta}+2 \mathrm{H} \partial_{\nu} \mathcal{V}_{3 \alpha} \mathcal{V}_{3 \beta}\right)-\frac{N_{c}}{12 \pi^{2}} \mu_{5}^{2} \omega^{\mu}, \\
& \left\langle\mathcal{J}_{3 A}^{\mu}\right\rangle_{\mathrm{cov}}=\frac{N_{c}}{48 \pi^{2}} \epsilon^{\mu \nu \alpha \beta}\left[3 \partial_{\nu} \mathcal{V}_{0 \alpha} \mathbb{T}_{\beta}-\partial_{\nu}\left(\mu_{5} u_{\alpha}\right) \mathbb{I}_{\beta}+\mu_{5} u_{\nu} \partial_{\alpha} \mathbb{I}_{\beta}\right] .
\end{aligned}
$$

The currents can now be decomposed into their longitudinal and transverse components with respect to the four-velocity $u_{\mu}$. The results can be written as linear combinations of the following five pseudo-scalar quantities

$$
\begin{aligned}
\mathbb{S}_{1, a} & \equiv \epsilon^{\mu \nu \alpha \beta} \mathbb{I}_{\mu} u_{\nu} \partial_{\alpha} \mathcal{V}_{a \beta}=\mathbb{I}_{\mu} \mathcal{B}_{a}^{\mu} \quad(a=0,3), \\
\mathbb{S}_{2} & \equiv \frac{1}{2} \epsilon^{\mu \nu \alpha \beta} \mathbb{I}_{\mu} u_{\nu} \partial_{\alpha} u_{\beta}=\mathbb{I}_{\mu} \omega^{\mu}, \\
\mathbb{S}_{3} & \equiv \epsilon^{\mu \nu \alpha \beta} u_{\mu}\left[\mathcal{V}_{3 \nu} \partial_{\alpha} \mathbb{I}_{\beta}-\frac{i}{3} \operatorname{Tr}\left(L_{\nu} L_{\alpha} L_{\beta}\right)\right], \\
\mathbb{S}_{4, a} & \equiv \epsilon^{\mu \nu \alpha \beta} \mathbb{T}_{\mu} u_{\nu} \partial_{\alpha} \mathcal{V}_{a \beta}=\mathbb{T}_{\mu} \mathcal{B}_{a}^{\mu} \quad(a=0,3), \\
\mathbb{S}_{5} & \equiv \frac{1}{2} \epsilon^{\mu \nu \alpha \beta} \mathbb{T}_{\mu} u_{\nu} \partial_{\alpha} u_{\beta}=\mathbb{T}_{\mu} \omega^{\mu},
\end{aligned}
$$

together with the four transverse pseudo-vectors

$$
\begin{array}{rlrl}
\mathbb{P}_{1, a}^{\mu} & \equiv \epsilon^{\mu \nu \alpha \beta} u_{\nu} \mathbb{I}_{\alpha} \partial_{\beta}\left(\frac{\mu_{a}}{T}\right) & (a=0,3), \\
\mathbb{P}_{2}^{\mu} & \equiv \epsilon^{\mu \nu \alpha \beta} u_{\nu} \partial_{\alpha} \mathbb{I}_{\beta}, & (a=0,3), \\
\mathbb{P}_{3, a}^{\mu} & \equiv \epsilon^{\mu \nu \alpha \beta} u_{\nu} \mathbb{T}_{\alpha} \partial_{\beta}\left(\frac{\mu_{a}}{T}\right) & \\
\mathbb{P}_{4}^{\mu} & \equiv \epsilon^{\mu \nu \alpha \beta} u_{\nu} \partial_{\alpha} \mathbb{T}_{\beta} .
\end{array}
$$

Notice that the definitions in eq. (5.23) involve either the magnetic field or the vorticity vector, defined in eqs. (3.16) and (3.17) respectively. The one-derivative corrections to the gauge currents can now be written as

$$
\begin{aligned}
u_{\mu}\left\langle\mathcal{J}_{0 V}^{\mu}\right\rangle_{\text {cov }} & =-\frac{N_{c}}{16 \pi^{2}}\left(\mathbb{S}_{1,3}+\mathbb{S}_{3}\right), \\
P_{\nu}^{\mu}\left\langle\mathcal{J}_{0 V}^{\nu}\right\rangle_{\text {cov }} & =-\frac{N_{c}}{16 \pi^{2}}\left(T \mathbb{P}_{1,3}^{\mu}-\mu_{3} \mathbb{P}_{2}^{\mu}-2 \mu_{5} \mathcal{B}_{0}^{\mu}\right),
\end{aligned}
$$

for the zero flavor components, while the three-component gives

$$
\begin{aligned}
u_{\mu}\left\langle\mathcal{J}_{3 V}^{\mu}\right\rangle_{\text {cov }} & =-\frac{N_{c}}{48 \pi^{2}}\left(3 \mathbb{S}_{1,0}+2 \mu_{5} \mathbb{S}_{5}\right), \\
P_{\nu}^{\mu}\left\langle\mathcal{J}_{3 V}^{\nu}\right\rangle_{\text {cov }} & =-\frac{N_{c}}{48 \pi^{2}}\left[3 T \mathbb{P}_{1,0}^{\mu}+\mu_{5} \mathbb{P}_{4}^{\mu}+4 \mu_{3} \mu_{5} \mathbb{H} \omega^{\mu}-2 \mu_{5}(\mathbb{H}+3) \mathcal{B}_{3}^{\mu}\right] .
\end{aligned}
$$

In all these equations, we have used that fields are time-independent (and in particular $A_{00}=-\mu_{5} u_{0}$ is constant), as well as the expression of the local temperature $T(\mathbf{x})=$ $T_{0} e^{-\sigma(\mathbf{x})}$. In addition, we applied the identity

$$
P^{\mu}{ }_{\nu} \epsilon^{\nu \omega \alpha \beta} \mathcal{T}_{\omega \alpha \beta}=-\epsilon^{\mu \nu \alpha \beta} u_{\nu} e^{-\sigma}\left(\mathcal{T}_{0 \alpha \beta}-\mathcal{T}_{\beta \alpha 0}\right),
$$


valid for any tensor $\mathcal{T}_{\mu \alpha \beta}$ transverse in the $\alpha$ index, $\mathcal{T}_{\mu \alpha \beta}=\mathcal{T}_{\mu \nu \beta} P_{\alpha}^{\nu}$. This equation can be applied when the last index in $\mathcal{T}_{\mu \alpha \beta}$ is also transverse, $\mathcal{T}_{\mu \alpha \beta}=\mathcal{T}_{\mu \nu \sigma} P_{\alpha}^{\nu} P_{\beta}^{\sigma}$, in which case the second term on the right-hand side of (5.27) is equal to zero.

We see how the covariant vector currents can be written solely in terms of the pseudoscalar structures $\mathbb{S}_{1, a}, \mathbb{S}_{3}$, and $\mathbb{S}_{5}$, as well as the pseudovectors $\mathbb{P}_{1, a}^{\mu}, \mathbb{P}_{2}^{\mu}$, and $\mathbb{P}_{4}^{\mu}$. The remaining structures, which will appear in the vector-axial currents, have zero transport coefficients associated. The contribution of the BZ terms to eqs. (5.25) and (5.26) can be easily identified as those terms proportional to the magnetic field $\mathcal{B}_{a}^{\mu}$ without gothic fonts prefactors [cf. (5.15)]. Of course, these terms can only appear in the transverse components of the covariant current, the BZ terms being themselves transverse. Notice as well that the expressions of the covariant currents are given only in terms of the KK-variant gauge fields $\mathcal{V}_{a \mu}$ and $\mathcal{A}_{0 \mu}$, without any explicit reference to the KK gauge field $a_{i}$.

Introducing the electric fields for $a=0,3$

$$
\mathcal{E}_{a \mu}=\mathcal{V}_{a \mu \nu} u^{\nu}=e^{-\sigma} \partial_{\mu} \mathcal{V}_{a 0}=T \partial_{\mu}\left(\frac{\mu_{a}}{T}\right)
$$

we can write the first pseudovector in (5.24) as

$$
\mathbb{P}_{1, a}^{\mu}=\frac{1}{T} \epsilon^{\mu \nu \alpha \beta} u_{\nu} \mathbb{I}_{\alpha} \mathcal{E}_{a \beta},
$$

and identify the terms proportional to $\mathbb{P}_{1,0}^{\mu}$ and $\mathbb{P}_{1,3}^{\mu}$ in the constitutive relations as the ones associated to the chiral electric effect [11]. In particular, we can construct the transverse covariant electromagnetic current as

$$
\begin{aligned}
P_{\nu}^{\mu}\left\langle\mathcal{J}_{\text {em }}^{\nu}\right\rangle_{\text {cov }}= & -\frac{e N_{c}}{48 \pi^{2}}\left[\epsilon^{\mu \nu \alpha \beta} u_{\nu} \mathbb{I}_{\alpha}\left(3 \mathcal{E}_{0 \beta}+\mathcal{E}_{3 \beta}\right)-\mu_{3} \epsilon^{\mu \nu \alpha \beta} u_{\nu} \partial_{\alpha} \mathbb{I}_{\beta}+\mu_{5} \epsilon^{\mu \nu \alpha \beta} u_{\nu} \partial_{\alpha} \mathbb{T}_{\beta}\right. \\
& \left.+4 \mu_{3} \mu_{5} \mathbb{H} \omega^{\mu}-2 \mu_{5} \mathbb{H}_{3}^{\mu}-2 \mu_{5}\left(\mathcal{B}_{0}^{\mu}+3 \mathcal{B}_{3}^{\mu}\right)\right] .
\end{aligned}
$$

Using (3.31) and (4.9), this can be written in terms of the electric $\mathcal{E}_{\mu}$, magnetic $\mathcal{B}_{\mu}$ and pions fields as

$$
\begin{aligned}
P_{\nu}^{\mu}\left\langle\mathcal{J}_{\text {em }}^{\nu}\right\rangle_{\text {cov }}= & \frac{e^{2} N_{c}}{12 \pi^{2} f_{\pi}} \epsilon^{\mu \nu \alpha \beta} u_{\nu} \partial_{\alpha} \pi^{0} \mathcal{E}_{\beta}-\frac{i \mu_{5} e N_{c}}{12 \pi^{2} f_{\pi}^{2}} \epsilon^{\mu \nu \alpha \beta} u_{\nu} \partial_{\alpha} \pi^{+} \partial_{\beta} \pi^{-} \\
& +\frac{\mu_{5} e^{2} N_{c}}{12 \pi^{2} f_{\pi}^{2}} \epsilon^{\mu \nu \alpha \beta} u_{\nu} \partial_{\alpha}\left(\pi^{+} \pi^{-}\right) \mathcal{V}_{\beta}+\frac{\mu_{3} \mu_{5} e N_{c}}{6 \pi^{2} f_{\pi}^{2}} \pi^{+} \pi^{-} \omega^{\mu} \\
& +\frac{5 \mu_{5} e^{2} N_{c}}{36 \pi^{2}} \mathcal{B}^{\mu}+\mathcal{O}\left(\pi^{3}\right) .
\end{aligned}
$$

Interestingly, the term proportional to $\pi^{+} \pi^{-} \mathcal{B}^{\mu}$ cancels out from this expression.

The emergence of the chiral electric effect, i.e. charge transport normal to the direction of the electric field, is manifest from the first term on eq. (5.31), with a transport coefficient that can be identified from this equation. Despite the ongoing discussion in the literature concerning its nondissipative character (see, for example, ref. [52]), our derivation of this term clearly shows that the chiral electric effect is intrinsically nondissipative, i.e. it does not lead to the production of entropy. Similar terms are present in the baryon and isospin currents. 
In fact, the fundamental feature preserving the nondissipative character of the chiral electric effect in the constitutive relation (5.31) is the appearance of the pion field having negative signature under time reversal $T$. Indeed, given the transformation properties of the pions, $\mathrm{T}: \pi^{0} \rightarrow-\pi^{0}, \mathrm{~T}: \pi^{ \pm} \rightarrow-\pi^{\mp}$, the corresponding transport coefficient must be T-even, thus revealing the reversible nature of the phenomenon. The situation is somewhat reminiscent of the quantum Hall effect, where nondissipative electric charge transport appears triggered by the presence of the T-odd external magnetic field. The case of the chiral hadronic fluid studied here, however, has a closer resemblance to the anomalous quantum Hall effect, where nondissipative transport is the result of T-breaking brought about by the nontrivial topology of the band [57].

To compare our results with other analysis in the literature, we use the identities (4.9) to write the spatial components of this current in terms of the pion fields and the electric and magnetic fields associated with the electromagnetic potential $\mathbb{V}_{\mu}$ as

$$
\begin{aligned}
\left\langle\mathcal{J}_{\mathrm{em}}^{i}\right\rangle_{\mathrm{cov}}= & \frac{e^{2} N_{c}}{12 \pi^{2} f_{\pi}} \epsilon^{i j k} \partial_{j} \pi^{0} \mathbb{E}_{k}-\frac{i \mu_{5} e N_{c}}{12 \pi^{2} f_{\pi}^{2}} \epsilon^{i j k} \partial_{j} \pi^{+} \partial_{k} \pi^{-} \\
& +\frac{\mu_{5} e^{2} N_{c}}{12 \pi^{2} f_{\pi}^{2}} \epsilon^{i j k} \partial_{j}\left(\pi^{+} \pi^{-}\right) \mathbb{V}_{k}+\frac{\mu \mu_{5} e N_{c}}{6 \pi^{2} f_{\pi}^{2}} \pi^{+} \pi^{-} \omega^{i} \\
& +\frac{5 \mu_{5} e^{2} N_{c}}{36 \pi^{2}}\left(\mathbb{B}^{i}-2 \mu \omega^{i}\right)+\mathcal{O}\left(\pi^{3}\right),
\end{aligned}
$$

where we have introduced the components of the physical electric field $\mathbb{E}_{i}=e^{-\sigma} \partial_{i} \bigvee_{0}$. In addition, we have introduced the electric charge chemical potential $\mu \equiv e^{-\sigma \bigvee_{0}}$ [cf. eqs. (3.27) and (4.12)]. This covariant electromagnetic current can be alternatively found by adding the corresponding $\mathrm{BZ}$ term to the consistent current obtained by varying the WZW equilibrium partition function [cf. (4.10)]

$$
\begin{aligned}
W\left[\mathbb{V}_{\mu}, \pi^{0}, \pi^{ \pm}\right]_{\mathrm{WZW}}= & -\int_{S^{3}} d^{3} x \sqrt{g}\left[\frac{e^{2} N_{c}}{12 \pi^{2} f_{\pi} T} \mu \partial_{k} \pi^{0}\left(\mathbb{B}^{k}-\mu \omega^{k}\right)\right. \\
& \left.+\frac{i \mu_{5} e N_{c}}{24 \pi^{2} f_{\pi}^{2} T}\left(\pi^{-} \partial_{k} \pi^{+}-\pi^{+} \partial_{k} \pi^{-}-2 i e \pi^{+} \pi^{-} \mathbb{V}_{k}\right)\left(\mathbb{B}^{k}-2 \mu \omega^{k}\right)\right]
\end{aligned}
$$

with respect to the electromagnetic vector potential $\mathbb{V}_{i}$. Similar expressions can be written for the baryon and isospin currents. In the first case, we find

$$
\left\langle\mathcal{J}_{\text {bar }}^{i}\right\rangle_{\mathrm{cov}}=\frac{e N_{c}}{12 \pi^{2}}\left[\frac{1}{f_{\pi}} \epsilon^{i j k} \partial_{j} \pi^{0} \mathbb{E}_{k}+\frac{1}{3} \mu_{5}\left(\mathbb{B}^{i}-2 \mu \omega^{i}\right)\right]+\mathcal{O}\left(\pi^{3}\right),
$$

while for the isospin current the result is

$$
\begin{gathered}
\left\langle\mathcal{J}_{\text {iso }}^{i}\right\rangle_{\mathrm{cov}}=-\frac{e N_{c}}{24 \pi^{2}}\left[-\frac{1}{f_{\pi}} \epsilon^{i j k} \partial_{j} \pi^{0} \mathbb{E}_{k}+\frac{2 i}{e f_{\pi}^{2}} \mu_{5} \epsilon^{i j k} \partial_{j} \pi^{+} \partial_{k} \pi^{-}-\frac{2}{f_{\pi}^{2}} \mu_{5} \epsilon^{i j k} \partial_{j}\left(\pi^{+} \pi^{-}\right) \mathbb{V}_{k}\right. \\
\left.-\frac{4}{f_{\pi}^{2}} \mu \mu_{5} \pi^{+} \pi^{-} \omega^{i}-3 \mu_{5}\left(\mathbb{B}^{i}-2 \mu \omega^{i}\right)\right]+\mathcal{O}\left(\pi^{3}\right) .
\end{gathered}
$$

All three currents are easily seen to be invariant under the gauge transformations of electromagnetism $\delta_{\varepsilon} \mathbb{V}_{i}=\partial_{i} \varepsilon, \delta_{\varepsilon} \pi^{ \pm}= \pm i e \varepsilon \pi^{ \pm}$. 
A relevant feature to notice in all three currents shown in eqs. (5.32), (5.34), and (5.35) is that the terms proportional to the vorticity vector $\omega^{i}$ come always multiplied by the chiral chemical potential $\mu_{5}$. This means that in the absence of chiral imbalance $\left(\mu_{5}=0\right)$ there are no contributions to the electromagnetic, isospin, and baryonic currents depending of the vorticity.

Despite of this fact, the partition function (5.33) shows the existence of a direct coupling of the vorticity vector to the pion gradient which is not weighted by $\mu_{5}$. Setting the electromagnetic field to zero for simplicity, this leads to the following form of the isospin number density

$$
n_{3}=\frac{(\operatorname{Tr} Q) e N_{c}}{2 \pi^{2} f_{\pi}} \mu \partial_{k} \pi^{0} \omega^{k},
$$

where we have written the result in terms of the trace of the charge matrix $Q$. This expression agrees with the results found in [58] for the baryon and isospin number densities of a hadronic fluid under rotation [59], which shows the existence of a vorticity coupling with the pion gradient in the absence of chiral imbalance. It is nevertheless surprising that no such effect survives in the spatial currents themselves, as it is seen from our explicit results.

To close this section, we study the one-derivative corrections to the constitutive relations derived from the covariant axial-vector currents. Their longitudinal and transverse projections are now given by

$$
\begin{aligned}
u_{\mu}\left\langle\mathcal{J}_{0 A}^{\mu}\right\rangle_{\mathrm{cov}} & =-\frac{N_{c}}{48 \pi^{2}} \mathbb{S}_{4,3}, \\
P_{\nu}^{\mu}\left\langle\mathcal{J}_{0 A}^{\nu}\right\rangle_{\mathrm{cov}} & =-\frac{N_{c}}{48 \pi^{2}}\left(T \mathbb{P}_{3,3}^{\mu}+2 \mu_{3} \mathbb{H}_{3}^{\mu}+4 \mu_{5}^{2} \omega^{\mu}\right),
\end{aligned}
$$

and

$$
\begin{aligned}
u_{\mu}\left\langle\mathcal{J}_{3 A}^{\mu}\right\rangle_{\mathrm{cov}} & =-\frac{N_{c}}{48 \pi^{2}}\left(3 \mathbb{S}_{4,0}-2 \mu_{5} \mathbb{S}_{2}\right) \\
P_{\nu}^{\mu}\left\langle\mathcal{J}_{3 A}^{\nu}\right\rangle_{\mathrm{cov}} & =-\frac{N_{c}}{48 \pi^{2}}\left(3 T \mathbb{P}_{3,0}^{\mu}+6 \mu_{3} \mathbb{H} \mathcal{B}_{0}^{\mu}-\mu_{5} \mathbb{P}_{2}^{\mu}\right) .
\end{aligned}
$$

As in the case of the vector currents, here we also find the occurrence of a nondissipative chiral electric effect associated with the terms $\mathbb{P}_{3, a}^{\mu}$, whose corresponding susceptibilities are obtained from the previous equations.

Expressed in terms of the KK-invariant electromagnetic fields, we find the following explicit expressions for the transverse components of the axial-vector covariant currents

$$
\begin{aligned}
\left\langle\mathcal{J}_{0 A}^{i}\right\rangle_{\mathrm{cov}}=\frac{N_{c}}{24 \pi^{2} f_{\pi}^{2}}\left[i e \epsilon^{i j k}\right. & \left(\pi^{-} \partial_{j} \pi^{+}-\pi^{+} \partial_{j} \pi^{-}-2 i e \pi^{+} \pi^{-} \mathbb{V}_{j}\right) \mathbb{E}_{k} \\
& \left.+2 e \mu \pi^{+} \pi^{-}\left(\mathbb{B}^{i}-2 \mu \omega^{i}\right)-2 \mu_{5}^{2} \omega^{i}\right]+\mathcal{O}\left(\pi^{3}\right), \\
\left\langle\mathcal{J}_{3 A}^{i}\right\rangle_{\mathrm{cov}}=\frac{N_{c}}{24 \pi^{2} f_{\pi}^{2}}\left[i e \epsilon^{i j k}\right. & \left(\pi^{-} \partial_{j} \pi^{+}-\pi^{+} \partial_{j} \pi^{-}-2 i e \pi^{+} \pi^{-} \mathbb{V}_{j}\right) \mathbb{E}_{k} \\
& \left.-2 e \pi^{+} \pi^{-}\left(\mathbb{B}^{i}-2 \mu \omega^{i}\right)\right]+\mathcal{O}\left(\pi^{3}\right) .
\end{aligned}
$$


These constitutive relations contain chiral separation effect terms of electric, magnetic, and vortical type. Although all explicit dependence on the vorticity in eq. (5.37) comes from the BZ term and seems to disappear in the absence of chiral imbalance $\left(\mu_{5}=0\right)$, once written in terms of the KK-invariant quantities we find vorticity-dependent terms in both constitutive relations, mediated by the charged pion fields, that survive in this limit.

Our calculation based on eq. (2.47) shows clearly that in the limit of vanishing NG fields, $\zeta_{a} \rightarrow 0(U \rightarrow \mathbb{1})$, the covariant currents are given by the BZ terms. For the electromagnetic transverse current, we find from eq. (5.32)

$$
\lim _{\pi^{0}, \pi^{ \pm} \rightarrow 0} P^{\mu}\left\langle\mathcal{J}_{\mathrm{em}}^{\nu}\right\rangle_{\mathrm{cov}}=\frac{5 \mu_{5} e^{2} N_{c}}{36 \pi^{2}} \mathcal{B}^{\mu}
$$

where the KK-variant magnetic field has been defined in eq. (3.32). This results exactly reproduces the transverse covariant electromagnetic current of the unbroken theory, as given in eq. (3.33). This contrasts with the Abelian model studied in ref. [60], in which the BZ term gives $\frac{1}{3}$ the result of the covariant current in the theory without spontaneous symmetry breaking.

\section{Closing remarks}

In this paper we have studied the partition function and anomalous currents of a two-flavor chiral hadronic (super)fluid using the expressions obtained in ref. [32] in a general setup, particularized to the background of interest. In a first instance, we focused our attention onto the unbroken theory, computing the partition function from the anomalous functional realizing the Bardeen form of the anomaly. Explicit forms of the covariant currents were provided in terms of the external sources, from where the transport coefficients associated with the magnetic and chiral vortical effect are obtained. The anomalous correction to the energy-momentum tensor of the unbroken theory was also computed.

In the case of the unbroken theory, we have explicitly computed the six transport coefficients in the constitutive relations for the vector and axial-vector covariant gauge currents, as well as the anomaly-induced corrections to the energy-momentum tensor. When expressed in terms of KK-variant fields, we find no vorticity-dependent terms in the constitutive relations for the vector currents. This is the result of the specific structure of the $\mathrm{U}(2) \times \mathrm{U}(2)$ group considered. Once written in terms of KK-invariant quantities, a nonvanishing chiral vortical conductivity appears, whose value is determined by the geometry of the static background metric.

For the theory with chiral symmetry breaking, our computation of the covariant currents and the constitutive relations avoids the use of the WZW effective action. Instead, we got the corresponding currents from the BZ terms of the unbroken theory, properly transformed by the matrix of NG bosons. Consistent currents were then easily evaluated by subtracting the contribution of the BZ polynomials. Our method greatly simplifies the calculation of consistent (and covariant) currents, since expressions evaluated on the particular background considered are used at every calculation step, instead of dealing with functional derivatives of the WZW action on a general background. 
While the functional derivatives of the partition function lead to the consistent currents in equilibrium, out-of-equilibrium expressions of those currents are retrieved by considering their Lorentz covariant generalizations (see, e.g. [37]). In our case, we proceeded by writing the covariant currents in a Lorentz covariant form, to retrieve the desired constitutive relations for the hydrodynamics of a relativistic fluid. We have used this method to find the constitutive relations in the chiral hadronic fluid, with and without spontaneous symmetry breaking. In the former case, they are expressed in terms of five pseudo-scalars and four pseudo-vectors quantities depending on the NG fields, together with the magnetic field and the vorticity vector. From them, we identify the pion field contributions to the chiral magnetic and vortical effects. Our calculation also predicts the emergence of a chiral electric effect whose corresponding transport coefficient is explicitly evaluated. This effect contributes to all vector and axial-vector covariant and consistent currents.

Whereas in the unbroken theory the chiral magnetic effect is restricted to the axialvector currents, when expressed in terms of KK-variant fields, we have found how the presence of NG bosons induces this effect also in vector currents. In all cases, its strength is controlled by the chemical potential governing chiral imbalance, and disappears in the limit $\mu_{5} \rightarrow 0$. A vorticity dependent term survives nonetheless in the number densities, agreeing with similar contributions found in ref. [58] using a different physical approach. As for the axial-vector global currents, they contain chiral separation effects associated with the electric and magnetic fields, as well as the vorticity. The later remains finite in the limit of vanishing chiral imbalance.

The strategy employed here to obtain the constitutive relations of a chiral (super)fluid can be extended to a wide class of theories with anomalous currents in both high energy and condensed matter physics. Gravitational and/or mixed gauge-gravitational anomalies [25] can also be incorporated into the description by considering the appropriate anomaly polynomials. Due to the presence of curvature terms, they would contribute at higher orders in the derivative expansion. Finding an economical and systematic way of computing the gravitational contributions to the constitutive relations is indeed of interest, given their recently discovered experimental signatures [61]. These and other issues will be addressed elsewhere.

\section{Acknowledgments}

We thank Claudio Corianò and Karl Landsteiner for discussions. The work of J.L.M. and M.V. has been supported by Spanish Science Ministry grant PGC2018-094626-B-C21 and Basque Government grant IT979-16. The research of E.M. is supported by Spanish MINEICO and European FEDER funds grants FIS2017-85053-C2-1-P, Junta de Andalucía grant FQM-225, and Consejería de Conocimiento, Investigación y Universidad of the Junta de Andalucía and European Regional Development Fund (ERDF) (grant No. SOMM17/6105/UGR), as well as by Universidad del País Vasco UPV/EHU through a Visiting Professor appointment and by Spanish MINEICO Ramón y Cajal Program (Grant No. RYC-2016-20678). M.A.V.-M. acknowledges the financial support from the Spanish Science Ministry through research grant PGC2018-094626-B-C22, as well as from Basque Government grant IT979-16. He also thanks the Department of Theoretical Physics of the University of the Basque Country for hospitality during the completion of this work. 
Open Access. This article is distributed under the terms of the Creative Commons Attribution License (CC-BY 4.0), which permits any use, distribution and reproduction in any medium, provided the original author(s) and source are credited.

\section{References}

[1] B. Zumino, Chiral anomalies and differential geometry, in Relativity, groups and topology, C. De Witt ed., Gordon adn Breach, U.K. (1983).

[2] L. Álvarez-Gaumé, An introduction to Anomalies, in Fundamental problems of gauge field theory, C. Velo and A.S. Wightman eds., Plenum Press, U.S.A. (1985).

[3] R.A. Bertlmann, Anomalies in quantum field theory, Oxford University Press, Oxford U.K. (1996).

[4] K. Fujikawa and H. Suzuki, Path integrals and quantum anomalies, Oxford University Press, Oxford U.K. (2004).

[5] J.A. Harvey, TASI 2003 lectures on anomalies, 2005, hep-th/0509097 [INSPIRE].

[6] A. Bilal, Lectures on anomalies, arXiv:0802.0634 [INSPIRE].

[7] D.T. Son and P. Surówka, Hydrodynamics with triangle anomalies, Phys. Rev. Lett. 103 (2009) 191601 [arXiv:0906.5044] [INSPIRE].

[8] K. Landsteiner, Notes on anomaly induced transport, Acta Phys. Polon. B 47 (2016) 2617 [arXiv: 1610.04413] [INSPIRE].

[9] K. Fukushima, D.E. Kharzeev and H.J. Warringa, The chiral magnetic effect, Phys. Rev. D 78 (2008) 074033 [arXiv:0808.3382] [INSPIRE].

[10] A.V. Sadofyev and M.V. Isachenkov, The chiral magnetic effect in hydrodynamical approach, Phys. Lett. B 697 (2011) 404 [arXiv:1010.1550] [INSPIRE].

[11] Y. Neiman and Y. Oz, Anomalies in superfluids and a chiral electric effect, JHEP 09 (2011) 011 [arXiv: 1106.3576] [INSPIRE].

[12] V.P. Kirilin, A.V. Sadofyev and V.I. Zakharov, Chiral vortical effect in superfluid, Phys. Rev. D 86 (2012) 025021 [arXiv:1203.6312] [InSPIRE].

[13] K. Fukushima, Views of the chiral magnetic effect, Lect. Notes Phys. 871 (2013) 241 [arXiv: 1209.5064] [INSPIRE].

[14] V.I. Zakharov, Chiral magnetic effect in hydrodynamic approximation, arXiv:1210.2186 [INSPIRE].

[15] D.E. Kharzeev and D.T. Son, Testing the chiral magnetic and chiral vortical effects in heavy ion collisions, Phys. Rev. Lett. 106 (2011) 062301 [arXiv: 1010.0038] [INSPIRE].

[16] M. Hongo, Y. Hirono and T. Hirano, Anomalous-hydrodynamic analysis of charge-dependent elliptic flow in heavy-ion collisions, Phys. Lett. B 775 (2017) 266 [arXiv:1309.2823] [INSPIRE].

[17] D.E. Kharzeev, J. Liao, S.A. Voloshin and G. Wang, Chiral magnetic and vortical effects in high-energy nuclear collisions - A status report, Prog. Part. Nucl. Phys. 88 (2016) 1 [arXiv: 1511.04050] [INSPIRE].

[18] X.-G. Huang, Electromagnetic fields and anomalous transports in heavy-ion collisions - A pedagogical review, Rept. Prog. Phys. 79 (2016) 076302 [arXiv:1509.04073] [InSPIRE]. 
[19] M. Kaminski, C.F. Uhlemann, M. Bleicher and J. Schaffner-Bielich, Anomalous hydrodynamics kicks neutron stars, Phys. Lett. B 760 (2016) 170 [arXiv:1410.3833] [INSPIRE].

[20] G. Sigl and N. Leite, Chiral magnetic effect in protoneutron stars and magnetic field spectral evolution, JCAP 01 (2016) 025 [arXiv:1507.04983] [INSPIRE].

[21] N. Yamamoto, Chiral transport of neutrinos in supernovae: Neutrino-induced fluid helicity and helical plasma instability, Phys. Rev. D 93 (2016) 065017 [arXiv:1511.00933] [INSPIRE].

[22] Y. Masada, K. Kotake, T. Takiwaki and N. Yamamoto, Chiral magnetohydrodynamic turbulence in core-collapse supernovae, Phys. Rev. D 98 (2018) 083018 [arXiv:1805.10419] [INSPIRE].

[23] S. Abbaslu, S.R. Zadeh and S.S. Gousheh, Contribution of the chiral vortical effect to the evolution of the hypermagnetic field and the matter-antimatter asymmetry in the early Universe, arXiv: 1908.10105 [INSPIRE].

[24] K. Jensen, R. Loganayagam and A. Yarom, Anomaly inflow and thermal equilibrium, JHEP 05 (2014) 134 [arXiv: 1310.7024] [INSPIRE].

[25] K. Jensen, R. Loganayagam and A. Yarom, Thermodynamics, gravitational anomalies and cones, JHEP 02 (2013) 088 [arXiv: 1207.5824] [INSPIRE].

[26] K. Jensen, R. Loganayagam and A. Yarom, Chern-Simons terms from thermal circles and anomalies, JHEP 05 (2014) 110 [arXiv:1311.2935] [INSPIRE].

[27] F.M. Haehl, R. Loganayagam and M. Rangamani, Effective actions for anomalous hydrodynamics, JHEP 03 (2014) 034 [arXiv: 1312.0610] [INSPIRE].

[28] G.M. Monteiro, A.G. Abanov and V.P. Nair, Hydrodynamics with gauge anomaly: variational principle and Hamiltonian formulation, Phys. Rev. D 91 (2015) 125033 [arXiv: 1410.4833] [INSPIRE].

[29] A. Jain, Galilean anomalies and their effect on hydrodynamics, Phys. Rev. D 93 (2016) 065007 [arXiv: 1509.05777] [INSPIRE].

[30] N. Banerjee, S. Dutta and A. Jain, Null fluids - A new viewpoint of galilean fluids, Phys. Rev. D 93 (2016) 105020 [arXiv:1509.04718] [InSPIRE].

[31] P. Glorioso, H. Liu and S. Rajagopal, Global anomalies, discrete symmetries and hydrodynamic effective actions, JHEP 01 (2019) 043 [arXiv: 1710.03768] [INSPIRE].

[32] J.L. Mañes, E. Megías, M. Valle and M.A. Vázquez-Mozo, Non-abelian anomalous (super)fluids in thermal equilibrium from differential geometry, JHEP 11 (2018) 076 [arXiv: 1806. 07647] [INSPIRE].

[33] K. Fukushima and K. Mameda, Wess-Zumino-Witten action and photons from the chiral magnetic effect, Phys. Rev. D 86 (2012) 071501 [arXiv:1206.3128] [INSPIRE].

[34] T. Brauner and H. Kolešová, Gauged Wess-Zumino terms for a general coset space, Nucl. Phys. B 945 (2019) 114676 [arXiv:1809.05310].

[35] R.M.A. Dantas, F. Peña-Benítez, B. Roy and P. Surówka, Non-Abelian anomalies in multi-Weyl semimetals, arXiv: 1905.02189 [INSPIRE].

[36] M. Nakahara, Geometry, topology and physics, $2^{\text {nd }}$ edition, Taylor \& Francis, U.K. (2003).

[37] N. Banerjee et al., Constraints on fluid dynamics from equilibrium partition functions, JHEP 09 (2012) 046 [arXiv: 1203.3544] [INSPIRE]. 
[38] J. Manes, R. Stora and B. Zumino, Algebraic study of chiral anomalies, Commun. Math. Phys. 102 (1985) 157 [INSPIRE].

[39] M. Giannotti and E. Mottola, The trace anomaly and massless scalar degrees of freedom in gravity, Phys. Rev. D 79 (2009) 045014 [arXiv:0812.0351] [INSPIRE].

[40] H.E. Haber and H.A. Weldon, Finite temperature symmetry breaking as Bose-Einstein condensation, Phys. Rev. D 25 (1982) 502 [INSPIRE].

[41] D. Yamada and L.G. Yaffe, Phase diagram of $N=4$ super-Yang-Mills theory with R-symmetry chemical potentials, JHEP 09 (2006) 027 [hep-th/0602074] [INSPIRE].

[42] R. Gatto and M. Ruggieri, Hot Quark Matter with an Axial Chemical Potential, Phys. Rev. D 85 (2012) 054013 [arXiv:1110.4904] [INSPIRE].

[43] X. Planells, A.A. Andrianov, V.A. Andrianov and D. Espriu, An effective theory for QCD with an axial chemical potential, PoS QFTHEP2013 (2013) 049 [arXiv:1310.4434] [INSPIRE].

[44] K. Landsteiner, E. Megías and F. Peña-Benítez, Gravitational anomaly and transport, Phys. Rev. Lett. 107 (2011) 021601 [arXiv:1103.5006] [INSPIRE].

[45] S. Golkar and D.T. Son, (Non)-renormalization of the chiral vortical effect coefficient, JHEP 02 (2015) 169 [arXiv:1207.5806] [inSPIRE].

[46] Y. Neiman and Y. Oz, Relativistic hydrodynamics with general anomalous Charges, JHEP 03 (2011) 023 [arXiv:1011.5107] [INSPIRE].

[47] K. Landsteiner, E. Megías and F. Peña-Benítez, Anomalous transport from Kubo formulae, Lect. Notes Phys. 871 (2013) 433 [arXiv:1207.5808] [INSPIRE].

[48] K. Jensen, P. Kovtun and A. Ritz, Chiral conductivities and effective field theory, JHEP 10 (2013) 186 [arXiv:1307.3234] [INSPIRE].

[49] E. Witten, Global aspects of current algebra, Nucl. Phys. B 223 (1983) 422 [InSPIRE].

[50] A. Andrianov, V. Andrianov and D. Espriu, Chiral imbalance in QCD, EPJ Web Conf. 138 (2017) 01007 [INSPIRE].

[51] S. Bhattacharyya, S. Jain, S. Minwalla and T. Sharma, Constraints on superfluid hydrodynamics from equilibrium partition functions, JHEP 01 (2013) 040 [arXiv:1206.6106] [INSPIRE].

[52] S. Chapman, C. Hoyos and Y. Oz, Superfluid Kubo formulas from partition function, JHEP 04 (2014) 186 [arXiv:1310.2247] [INSPIRE].

[53] P. Kovtun, Lectures on hydrodynamic fluctuations in relativistic theories, J. Phys. A 45 (2012) 473001 [arXiv: 1205. 5040] [INSPIRE].

[54] L. Rezzolla and O. Zanotti, Relativistic hydrodynamics, Oxford University Press, Oxford U.K. (2013).

[55] C.G. Callan Jr. and J.A. Harvey, Anomalies and Fermion zero modes on strings and domain walls, Nucl. Phys. B 250 (1985) 427 [INSPIRE].

[56] S. Iso, H. Umetsu and F. Wilczek, Hawking radiation from charged black holes via gauge and gravitational anomalies, Phys. Rev. Lett. 96 (2006) 151302 [hep-th/0602146] [INSPIRE].

[57] F.D.M. Haldane, Model for a quantum Hall effect without Landau levels: condensed-matter realization of the 'parity anomaly', Phys. Rev. Lett. 61 (1988) 2015 [INSPIRE]. 
[58] X.-G. Huang, K. Nishimura and N. Yamamoto, Anomalous effects of dense matter under rotation, JHEP 02 (2018) 069 [arXiv:1711.02190] [INSPIRE].

[59] K. Fukushima, Extreme matter in electromagnetic fields and rotation, Prog. Part. Nucl. Phys. 107 (2019) 167 [arXiv: 1812.08886] [INSPIRE].

[60] I. Amado, N. Lisker and A. Yarom, Universal chiral conductivities for low temperature holographic superfluids, JHEP 06 (2014) 084 [arXiv:1401.5795] [INSPIRE].

[61] J. Gooth et al., Experimental signatures of the mixed axial-gravitational anomaly in the Weyl semimetal NbP, Nature 547 (2017) 324 [arXiv:1703.10682] [INSPIRE]. 